\title{
Computational analysis of personality and emotion in semi-structured interviews
}

\author{
by
}

Vidya David

A thesis submitted to the Faculty of Graduate and Postdoctoral Affairs in partial fulfillment of the requirements for the degree of

Master of Arts

in

Cognitive Science

Carleton University

Ottawa, Canada

(C) 2017 Vidya David 


\begin{abstract}
Psychopathy is a personality disorder involving deficits in affective characteristics and behaviour (Cleckley, 1988; Hare, 2003). Previous studies have found relationships between psychopathy and negative polarity in text, as well as psychopathy and specific semantic content (e.g., Body, Family) (Garcia \& Sikström, 2014; Hancock, Woodworth, \& Porter, 2013; Sumner, Byers, Boochever, \& Park, 2012). The majority of these studies were performed with non-clinical psychopathy (from the general population), and the only study on clinical psychopathy (from institutionalized populations) failed to find support for a relationship between overall psychopathy and negative polarity (Hancock et al., 2013). The current study explores emotion and semantic categories in further detail with both a non-clinical and a clinical sample. Findings were inconsistent with the majority of previous research, suggesting that linguistic correlates of psychopathy are variable. The prevalence of such correlates is possibly dependent on sample size and text source.
\end{abstract}




\section{Acknowledgments}

I would like to thank my supervisor, Dr. John Logan, for his continuous guidance and encouragement while completing my thesis project. Thank you to my co-supervisor, Dr. Diana Inkpen for her assistance, especially with the subject of text mining, and for being so accessible. Thank you to my committee member Dr. Kasia Muldner, for her advice and support. I would also like to thank Dr. Ida Toivonen for her help with data collection, and Liane Dubreuil for her understanding and guidance with administration. I would like to thank my friends and family who pushed me when I needed motivation, and supported me when I needed reassurance. Finally, this research was supported by the Social Sciences and Humanities Research Council of Canada and would not have been possible without funding from the council. 
Table of Contents

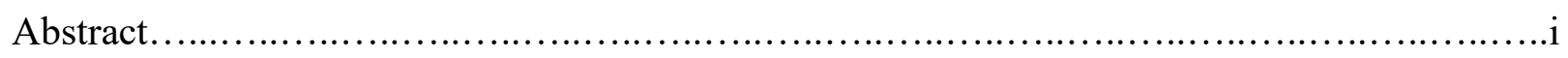

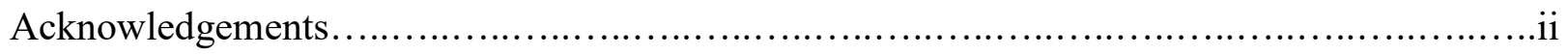

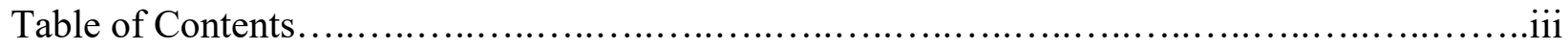

List of Tables............................................................................. vi

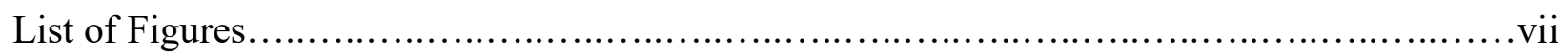

List of Appendices....................................................................

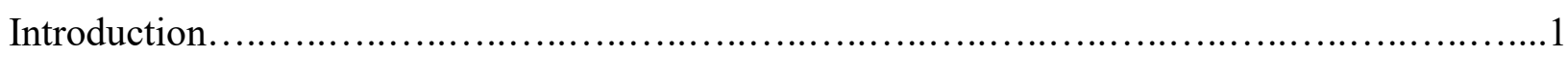

Psychopathy......................................................................

Psychopathy Subtypes.......................................................

Theories of Non-Clinical Psychopathy...................................... 4

Psychopathy Measures.......................................................6

Clinical Psychopathy Measures.............................................6

Non-Clinical Psychopathy Measures....................................7

Psychopathy and Emotion..........................................................

Emotion Perception.......................................................... 9

Emotion Production.................................................................11

Discussion...................................................................... 12

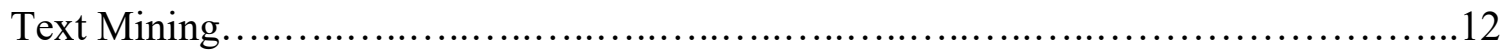

Sentiment Analysis.................................................... 13

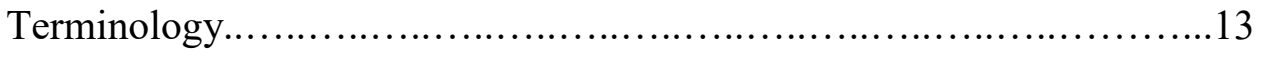

Lexicon-based Sentiment Analysis...................................15

Machine Learning Sentiment Analysis.................................17

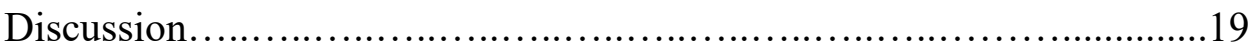

Semantic Analysis...........................................................20

Closed-vocabulary Analysis.........................................20

Open-vocabulary Analysis..........................................22 


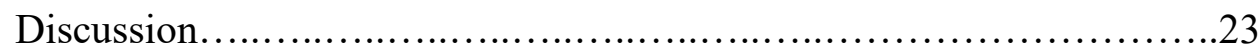

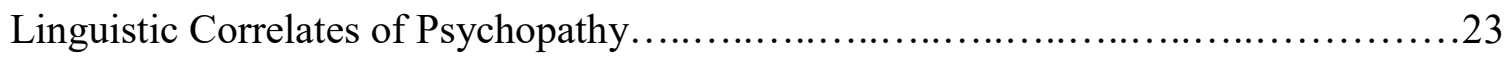

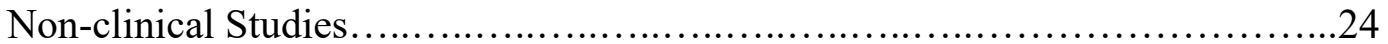

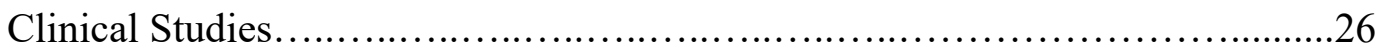

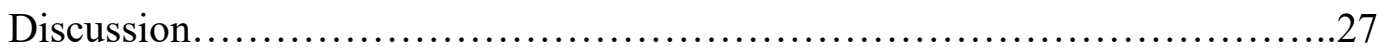

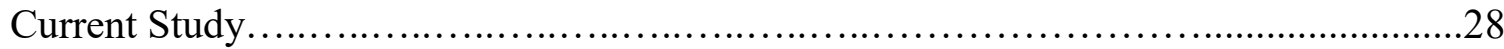

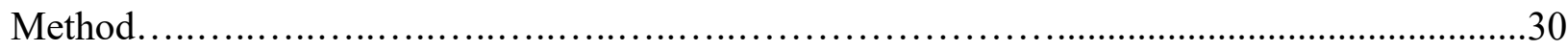

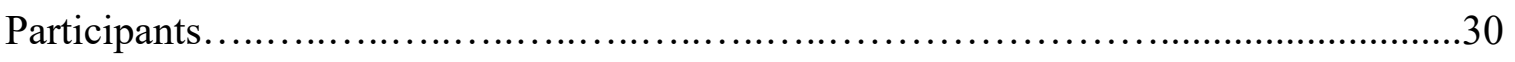

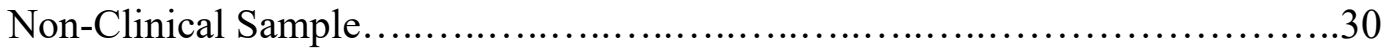

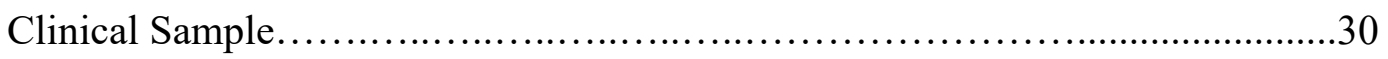

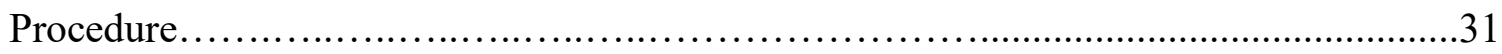

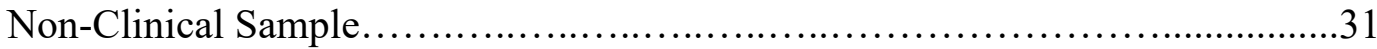

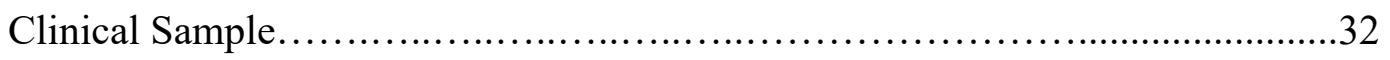

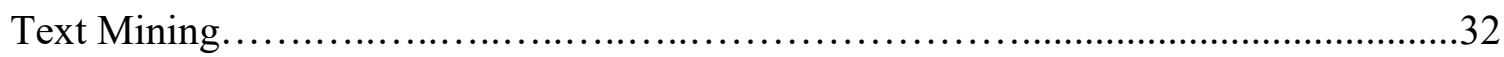

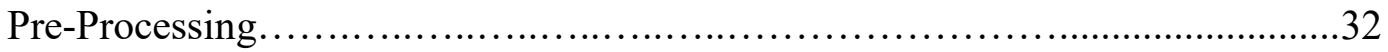

Sentiment and Semantic Analysis.............................................33

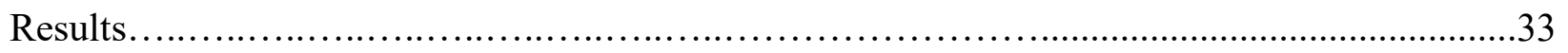

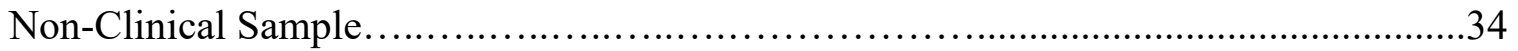

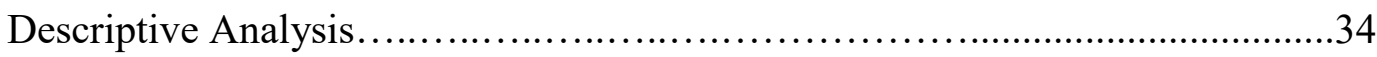

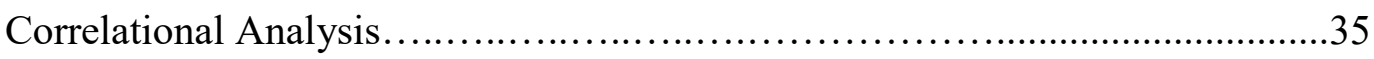

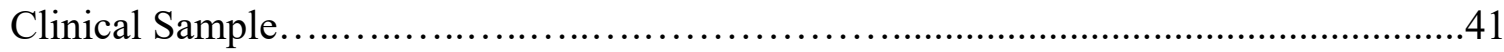

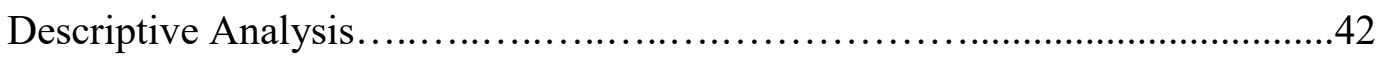

Correlational Analysis................................................................42

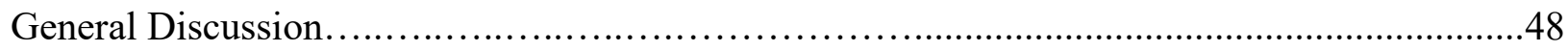

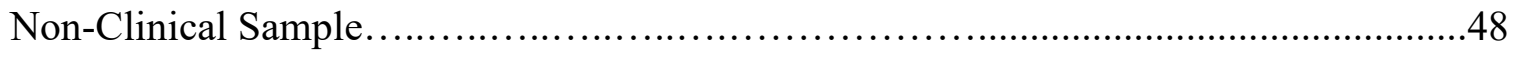

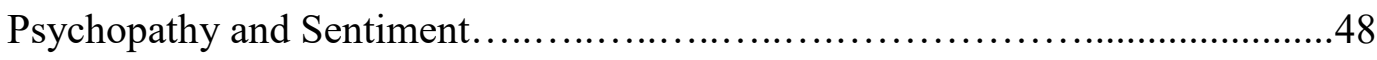

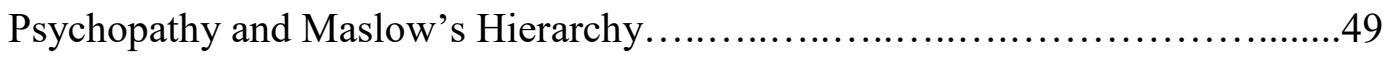

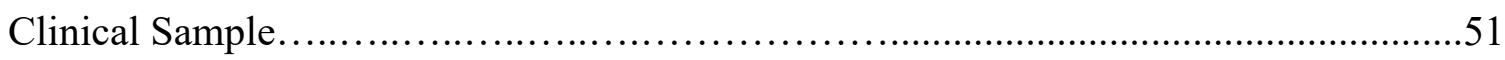




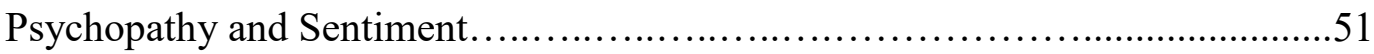

Psychopathy and Maslow's Hierarchy....................................52

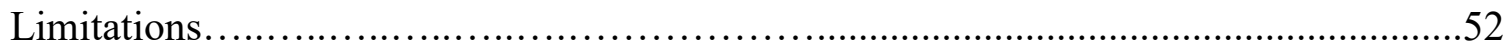

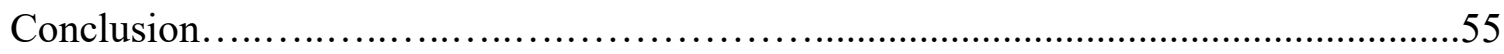

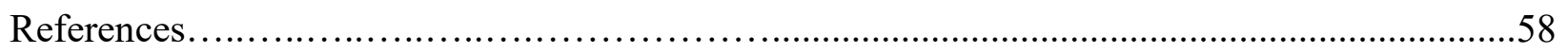




\section{List of Tables}

Table 1: Mean $(M)$, standard deviation $(S D)$, skew, kurtosis, and Shapiro-Wilk statistic and its pvalue for linguistic features and non-clinical psychopathy scores...................................36

Table 2: Correlation coefficients $(r)$ and p-values $(p)$ for two-tailed bivariate Pearson correlation between psychopathy measures and linguistic features for non-clinical data, $*=$ significant at $\alpha=0.05, * *=$ significant at $\alpha=0.002$.

Table 3: Correlation coefficients $(r)$ and p-values $(p)$ for two-tailed bivariate Pearson correlation between psychopathy measures and linguistic features for non-clinical data excluding univariate outliers, $*=$ significant at $\alpha=0.05, * *=$ significant at $\alpha=0.002 \ldots \ldots \ldots \ldots \ldots \ldots \ldots . . . .38$

Table 4: Correlation coefficients $(r)$ and p-values $(p)$ for two-tailed bivariate Pearson correlation between psychopathy measures and linguistic features for non-clinical data excluding bivariate outliers, $*=$ significant at $\alpha=0.05, * *=$ significant at $\alpha=0.002$.

Table 5: Correlation coefficients $(r)$ and p-values $(p)$ for two-tailed bivariate Pearson correlation between psychopathy measures and linguistic features for non-clinical data with swear words included, $*=$ significant at $\alpha=0.05, * *=$ significant at $\alpha=0.002$.

Table 6: Correlation coefficients $(r)$ and p-values $(p)$ for two-tailed bivariate Pearson correlation between psychopathy measures and linguistic features for non-clinical data using LIWC, * $=$ significant at $\alpha=0.05, * *=$ significant at $\alpha=0.002$.

Table 7: Mean $(M)$, standard deviation $(S D)$, skew, kurtosis, and Shapiro-Wilk statistic and its pvalue for linguistic features and clinical psychopathy scores.....

Table 8: Correlation coefficients $(r)$ and p-values $(p)$ for two-tailed bivariate Pearson correlation between psychopathy measures and linguistic features for clinical data, $*=$ significant at $\alpha=0.05, * *=$ significant at $\alpha=0.002$

Table 9: Correlation coefficients $(r)$ and p-values $(p)$ for two-tailed bivariate Pearson correlation between psychopathy measures and linguistic features for clinical data excluding univariate outliers, $*=$ significant at $\alpha=0.05, * *=$ significant at $\alpha=0.002$.

Table 10: Correlation coefficients $(r)$ and p-values $(p)$ for two-tailed bivariate Pearson correlation between psychopathy measures and linguistic features for clinical data with swear words included, $*=$ significant at $\alpha=0.05, * *=$ significant at $\alpha=0.002$.

Table 11: Correlation coefficients $(r)$ and p-values $(p)$ for two-tailed bivariate Pearson correlation between psychopathy measures and linguistic features for clinical data using LIWC, $*=$ significant at $\alpha=0.05, * *=$ significant at $\alpha=0.002$. 


\section{List of Figures}

Figure 1: Boxplots for a) psychopathy scales and b) semantic features for non-clinical data with

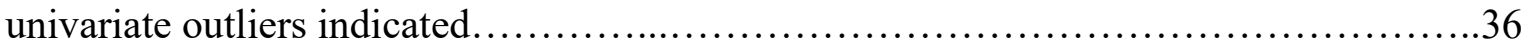

Figure 2: Scatterplot matrix of psychopathy scales and semantic features for non-clinical data with bivariate outliers indicated....................................................

Figure 3: Scatterplot matrix of psychopathy scales and semantic features for non-clinical data

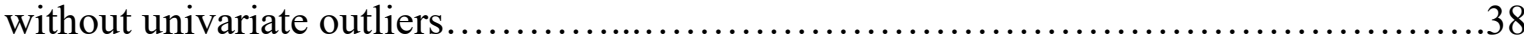

Figure 4: Boxplots for a) psychopathy scales and b) semantic features for clinical data with

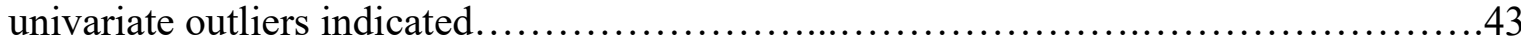

Figure 5: Scatterplot matrix of psychopathy scales and semantic features for clinical data with

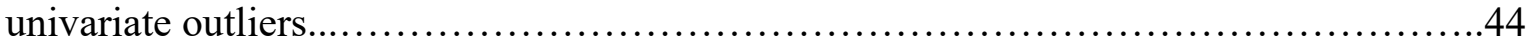

Figure 6: Scatterplot matrix of psychopathy scales and semantic features for clinical data without

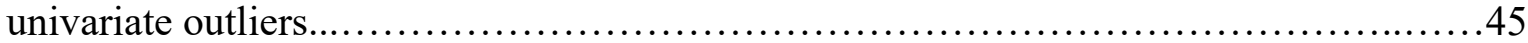




\section{List of Appendices}

Appendix A: Interview Questions................................................ 76

Appendix B: SRP-III-SF Questionnaire............................................... 79 


\section{Computational Analysis of Personality and Emotion in Semi-structured Interviews}

Language provides a way of examining how an individual represents information in memory and how that information is accessed and utilized. The psychological construct of personality, described as the stable formation of a person's thoughts, feelings and behaviours (Funder, 2007; Teo, 2014), is closely related to language usage (Hirsh \& Peterson, 2009; Yarkoni, 2010). The majority of studies on language and personality have looked at the relation between linguistic features and non-disordered personality traits. However, a relation between language and personality disorders is also possible since personality disorders may be viewed as extreme expressions of general personality traits (Samuel \& Widiger, 2008; O’Connor, 2005). Personality disorders are defined as persisting and dysfunctional patterns of behaviour which significantly disrupt an individual's everyday life (Martin, 2015).

The relation between personality disorders and general personality traits is especially prevalent in psychopathy (Guay, Ruscio, Knight, \& Hare, 2007). Psychopathy is a personality disorder with deficits in emotion processing and interpersonal interactions, and which has both clinical and non-clinical variants (Lebreton, Binnng, \& Adorno, 2006). Given this relationship, it is likely that psychopathy can also be predicted using linguistic features. Few studies have previously explored the possible relationship between language and psychopathy (Garcia \& Sikström, 2014; Hancock, Woodworth, \& Porter, 2013; Preotiuc-Pietro, Carpenter, Giorgi, \& Ungar, 2016; Sumner, Byers, Boochever, \& Park, 2012; Wald, Khoshgoftaar, \& Napolitano, 2012). Results from these studies indicate that the emotional polarity (e.g., positive vs. negative) and semantic content of language may be particularly useful for predicting psychopathy. However, these studies are mainly of non-clinical psychopaths, not clinical psychopaths. In addition, the majority fail to use detailed emotional polarity information. 
In the current study, I look at detailed emotional polarity and semantic content as predictors of both non-clinical and clinical psychopathy. My study contributes to the understanding of psychopathy and language. Such research informs future work on using language to predict psychopathy or differentiate clinical and non-clinical psychopathy. The latter would be particularly useful as clinical psychopathy is associated with more violent and frequent crimes (Hare, 2003; Leistico, Salekin, DeCoster, \& Rogers, 2008). In addition, this work contributes to the understanding of emotion production in psychopathy, including clinical psychopathy, since very few studies have explored this subject, and previous results have been variable (Hancock et al., 2013).

\section{Psychopathy}

Psychopathy is defined as a personality disorder involving deficits in emotion, interpersonal traits, and behaviour. It is noticeably characterized by a manipulative personality and a lack of empathy (Blair, Mitchell, \& Blair, 2005; Cleckley, 1988). Modern conceptualizations of psychopathy are based on the seminal work of Cleckley $(1941 ; 1976)$ and the Psychopathy Checklist (PCL) scale created by Hare (1980). Cleckley (1941) outlined 16 criteria of what he believed described psychopathy from his case studies with patients in a psychiatric hospital. These criteria include superficial charm, egocentricity, failure to form lasting intimate relationships, etc. (Cleckley, 1976). In the 1970s, Hare created the PCL based on these criteria, as well as criteria based on Hare's own encounters with individuals in both psychiatric hospitals and prisons (Blair et al., 2005). The most popular version of the PCL is the Psychopathy Checklist-Revised (PCL-R, Hare, 1991, 2003). The PCL-R contains 20 items, many of which have similarities with Cleckley's (1941) original 16 criteria. 
Significant intercorrelations exist between the 20 PCL-R items, suggesting the presence of an underlying concept, which is assumed to be psychopathy (Neumann, Hare, \& Newman, 2007). In addition, stronger intercorrelations exist between specific items, creating subgroups among the items. For example, in the PCL-R, 18 of the 20 items are categorized into four interrelated facets: Interpersonal items, Affective items, Lifestyle items, and Antisocial items. In turn, these four facets can be grouped into two broad correlated factors. Factor 1 contains interpersonal/affect items while Factor 2 contains lifestyle/antisocial items (Hare, 2003). This two-factor model has shown to exist in large samples of participants (Brandt, Kennedy, Patrick, \& Curtin, 1997; Hare \& Neumann, 2006; Neumann et al., 2007), and has been supported by other versions of the PCL, including the Psychopathy Checklist- Youth Version (PCL-YV; Forth \& Kosson, 2003).

Psychopathy has traditionally been interpreted as a taxonomic construct where individuals are classified as being either psychopaths or non-psychopaths (Guay et al., 2007; Hare, 1980). However, recent evidence suggests that a dimensional interpretation of psychopathy may be more appropriate for describing the disorder, where individuals are merely closer or farther from the prototypical psychopath (Edens et al., 2006; Guay et al., 2007). These studies analyzed the scores given to individuals from a variety of psychopathy scales and found that a dimensional structure of psychopathy is consistently supported and not a taxonomic one. Given this trend, I adopt a dimensional interpretation of psychopathy for the present study.

Psychopathy Subtypes. Psychopathy is described as consisting of two variants: clinical and non-clinical psychopathy. Clinical psychopathy, also known as criminal psychopathy, refers to individuals whose behaviour causes significant harm to others and themselves. Due to emotional and behavioural deficits, they have problems functioning in everyday situations (Lebreton et al., 
2006). For example, clinical psychopathy is strongly correlated with a high risk of violence and risk in correctional settings (Hare, 2003; Leistico et al., 2008). For this reason, clinical psychopaths are often found in institutions such as prisons or psychiatric hospitals. Nonetheless, the presence of clinical psychopathy is very low, with only a $1 \%$ present in the total population (Lebreton et al., 2006).

The second subtype of psychopathy is non-clinical psychopathy, also referred to as nonclinical or non-institutionalized psychopathy as well as sub-clinical psychopathy. Non-clinical psychopaths are found in the general population and are characterized as having the same traits as clinical psychopaths, but with more limited interactions with the criminal justice system (Hall \& Benning, 2006; Widom, 1977). Some have suggested that non-clinical psychopaths instead express antisocial behaviour through more "socially-acceptable" means, such as social, ethical, or professional venues. Therefore, while still harmful towards others, this behaviour is not illegal and therefore not deemed clinically impairing (Hall \& Benning, 2006; Smith, 1978; Widom, 1977). For example, non-clinical psychopathy is associated with minor criminal or non-illegal infractions such as substance abuse, relational aggression, and sexual harassment (Benning et al., 2003; Gustafson \& Ritzer, 1995; LeBreton et al., 2006). In addition, compared to a community sample, psychopathic traits were more prevalent in corporate individuals (Babiak \& Hare, 2007). This suggests that non-clinical psychopathy may be beneficial rather than detrimental to individuals in certain circumstances where non-ethical and non-social behaviour are advantageous (Babiak \& Hare, 2007; Lykken, 1995). Non-clinical psychopathy is slightly more prevalent than clinical psychopathy, at $\sim 5-10 \%$ of the total population.

Theories of Non-Clinical Psychopathy. Few studies have explicitly studied the difference between clinical and non-clinical psychopathy (e.g., Hall \& Benning, 2006, LeBreton et al., 
2006). According to Hall and Benning (2006), three main theories have been brought forward in the literature. It should be noted that while these theories each have a unique perspective, they are not mutually exclusive, and it may be that all contribute in some way to our understanding of the variants of psychopathy. The theories provide different explanations of non-clinical psychopathy and its relation to clinical psychopathy.

The first theory suggests that non-clinical psychopathy is a sub-clinical expression of clinical psychopathy. That is, it postulates that non-clinical psychopaths have the same deficits as clinical psychopaths, but that they display them to a lesser degree of severity and frequency (Gustafson \& Ritzer, 1995; Pethman \& Erlandsson, 2002). This is supported by the findings that non-clinical psychopathy is associated with infrequent and minor or non-illegal infractions (Benning et al., 2003; Gustafson \& Ritzer, 1995). In addition, this sub-clinical theory can also be interpreted to support the view that psychopathy is dimensional as it assumes that non-clinical and clinical psychopathy are based on the same underlying construct.

The second theory suggests that non-clinical psychopathy is moderated by environmental factors. Therefore, it assumes that non-clinical psychopaths originally have the same deficits as well as the same frequency and severity of deficit expressions as clinical psychopaths, but that in non-clinical psychopaths these expressions are moderated by a variety of environmental factors (Lykken, 1995). These factors, such as intelligence, education, parental supervision and socioeconomic status, are believed to prevent psychopathic traits from being expressed in clinical form. For example, correlations have been found between clinical psychopathy and low intelligence, lack of early parental supervision, and low familial socioeconomic status (Farrington, 2006). In addition, correlations were found between non-clinical psychopathy and parental supervision as well as with family socioeconomic status (Mahmut et al., 2008). 
Finally, the third theory hypothesizes that non-clinical psychopathy is an expression of only Factor 1 psychopathic traits. Therefore, according to this theory, non-clinical and clinical psychopathy involve different deficits, and therefore are etiologically distinct. Specifically, nonclinical psychopaths having predominant deficits in Factor 1 traits and less severe deficits in Factor 2 traits (Patrick, 2001). In comparison, clinical psychopaths have severe deficits in both factors. Non-clinical psychopaths have higher Factor 1 deficits than Factor 2 deficits in many studies (DeMatteo, Heilbrun, \& Marczyk, 2006; Levenson, 1992; Lilienfeld, 1994).

Psychopathy Measures. Several measures of psychopathy exist for both clinical and nonclinical psychopathy. These include measures that were specifically created for psychopathy or found to have correlations with psychopathy. The most popular measures will be described here.

Clinical Psychopathy Measures. For clinical psychopathy, the most popular scale continues to be the PCL-R. In the PCL-R, a clinician or researcher interviews an individual and also reviews their case file, scoring them on each of the 20 PCL-R items (Hare, 2003). The individual is measured on each of the four facets of the PCL-R as well as a total PCL-R score ranging from 0 to 40 . In research and clinical settings, a cut-off score of 30 is often used (Hare \& Neumann, 2008). However, as mentioned earlier, a dimensional interpretation of the scores may also be used, where the scores are viewed on a continuous scale. The reliability and validity of the PCLR has been supported by many studies (Bolt, Hare, \& Neumann, 2007; Cooke, Michie, Hart, \& Clark, 2005; Hare, 2003). For this reason, the PCL-R remains the gold standard for measuring psychopathy in clinical populations. The PCL-R has been modified to create alternate versions, including a shorter form of the measure to make the Psychopathy Checklist Screening Version (PCL:SV; Hart, Cox, \& Hare, 1995) and a version designed for adolescents, the Psychopathy Checklist Youth Version (PCL-YV; Forth, Kosson, \& Hare, 2003). 
Non-Clinical Psychopathy Measures. In terms of non-clinical psychopathy, a larger variety of measures exist, partially due to a recent growing interest in non-clinical psychopathy. These include: The Self -Report Psychopathy scale (SRP-III; Paulhus, Hemphill, \& Hare, 2012); the Dirty Dozen questionnaire (Jonason \& Webster, 2010), Short Dark Triad questionnaire (SD3; Jones \& Paulhus, 2014); NEO Personality Inventory - Revised (NEO PI-R; Costa \& McCrae, 1992); and Eysenck Personality Questionnaire - Revised (EPQ-R; Eysenck \& Eysenck, 1991).

One of the most well-supported scales used to measure non-clinical psychopathy is the SRPIII (Paulhus, Hemphill, \& Hare, 2012). The scale takes the form of a 64-item self-report questionnaire. For each item, individuals indicate how much they agree or disagree with a statement. The scale has four subscales which relate to the four dimensions of the PCL-R. Therefore, it can be used to compare both overall score and factor score between clinical and non-clinical psychopath populations. Many studies have confirmed the reliability and validity of this scale in a variety of populations (e.g., Williams, Paulhus, \& Hare, 2007; Watt \& Brooks, 2012; Neal \& Sellbom, 2012; Mahmut, Menictas, Stevenson, Stevenson, \& Homewood, 2011).

Paulhus and Williams (2002) have recently suggested a relation between non-clinical psychopathy and sub-clinical narcissism and Machiavellianism. Together, all three are referred to as the Dark Triad of personality. Paulhus and Williams (2002) suggested that grouping these socially negative personality types together may lead to a better understanding of them individually. In addition, research has explored the relation between non-clinical psychopathy and general personality traits (Guay et al., 2007; Derefinko \& Lynam, 2013; Lynam, 2002). As a consequence of these lines of research, studies on non-clinical psychopathy have used measures which are based on the PCL-R itself, on the Dark Triad concept, as well as general personality traits. To measure non-clinical psychopathy in the context of the Dark Triad, two scales are 
popularly used. These are the Dirty Dozen questionnaire (Jonason \& Webster, 2010) and SD3 (Jones \& Paulhus, 2014) Likert-scale self-report questionnaires. The Dirty Dozen questionnaire contains only 12 items, with 4 items per personality type, resulting in criticisms of its use (Lee et al., 2013). The SD3 was designed to be brief, but more valid. The SD3 contains 27 items, with 9 items per personality type. It has more validity in comparison to the Dirty Dozen questionnaire (Maples, Lamkin, \& Miller, 2014). Despite this difference, the Dirty Dozen is still often used to measure non-clinical psychopathy due to its extreme brevity (e.g., Preotiuc-Pietro et al., 2016). However, due to the structure of both questionnaires, neither describe non-clinical psychopathy in terms of the two-factor model. This prevents comparison of non-clinical psychopathy studies which use these Dark Triad questionnaires and clinical psychopathy studies which use the PCLR.

In relation to general personality traits, non-clinical psychopathy has been described both using the popular Five-Factor model of personality (FFM; Digman, 1990; Tupes \& Christal, 1992) as well as the older three-factor theory of personality by Eysenck (Eysenck, 1952). The FFM is often measured using the NEO PI-R (Costa \& McCrae, 1992), while Eysenck's theory of personality is measured using the EPQ-R (Eysenck \& Eysenck, 1991). The NEO PI-R contains 30 items with 6 items for each of the five personality traits: Extraversion, Neuroticism, Openness to experience, Agreeableness, and Conscientiousness. Overall, a majority of studies have formed the consensus that psychopathy is associated with high scores on Extraversion and low scores on Neuroticism, Openness, Agreeableness and Conscientiousness, although specific trait items may correlate differently (Derefinko \& Lynam, 2013; Harpur, Hart, \& Hare, 2002). Similarly, Eysenck's theory of personality outlines three traits: Neuroticism, Extraversion, and Psychoticism. Although some researchers have found that psychopathy is associated with a high 
score on one of these traits, Psychoticism (e.g., Zuckerman, 1989), others have found a lack of correlation between Psychoticism and the original PCL (Hare, 1982). No relationship has yet been established between the EPQR and SRP-III, which may be viewed as the gold standard measurement of non-clinical psychopathy. In contrast, FFM traits have been correlated with the PCL, although the correlated traits differed depending on whether the sample was from a clinical or non-clinical population (Hart \& Hare, 1994). Specifically, in clinical psychopaths, PCL scores were significantly related to low Agreeableness and Conscientiousness while in non-clinical psychopaths, they were only related to low Agreeableness. In addition, Widiger and Lynam (1998) suggested that FFM facets could also be used to describe the two-factor model of psychopathy, allowing comparison with studies that use the PCL-R. No relation has yet been established between Eysenck's theory and the PCL-R's two-factor structure.

\section{Psychopathy and Emotion}

Emotion impairments in psychopathy have been known to exist from Cleckley’s (1941) initial descriptions of the personality disorder. They are believed to be an integral component of the disorder. The majority of findings related to psychopathy and emotion however have been in terms of the perception of emotion by individuals with psychopathic characteristics. Few studies have looked at the production of emotion.

Emotion Perception. Emotion perception is known to be significantly impaired in psychopathy. The most prominent and consistent deficits have been found in facial affect recognition, vocal affect recognition, lexical priming, and startle reflex response (see Brook, Brieman, \& Kosson 2013, for a review). Overall, studies have found that psychopaths demonstrate reduced processing of emotion information compared to non-psychopaths. These results have been demonstrated in both clinical and non-clinical populations (Blair et al., 2006; 
Long \& Titone, 2007). For example, incarcerated male clinical psychopaths had a slower response to emotionally related words (e.g., "song" and "love") than to semantically related words (e.g., "dog" and "cat"). In addition, they had a slower response to words that were emotionally related compared to clinical non-psychopaths (Blair et al., 2006). Similarly, Long and Titone (2007) found that student male non-clinical psychopaths were less efficient in responding to processing negatively valanced words than non-clinical non-psychopaths.

A division exists in research on emotion perception in psychopathy regarding whether all emotions are impaired (Cleckley, 1941), or only negative emotions are impaired, specifically fear and sadness (Lykken, 1995). Regarding these theories, the literature shows conflicting conclusions, or the finding that neither theory has substantial support (Brook et al., 2013; Dawel, O’Kearney, McKone, \& Palermo, 2012; Wilson, Juodis, \& Porter, 2011). For example, Wilson et al. (2011) performed a meta-analysis on 22 facial affect recognition studies in psychopathy studies and found that overall, correlations were small between facial affect recognition and impairments in all emotions (i.e. positive and negative emotions). Strong correlations, however, were found between facial affect recognition deficits and impairments in fear and sadness. Conversely, Dawel et al. (2012) reviewed and analyzed 26 studies of both facial and vocal affect recognition in psychopathy and found correlations between facial affect recognition and deficits in all emotions, even when fear and sadness were removed from the model. This did not hold for vocal affect recognition where correlations between affect recognition and deficits in all emotion lost their significance when fear and sadness were removed. This suggests psychopaths were impaired in all emotions for recognizing affect in faces, but only impaired in fear and sadness for recognizing vocal affect. Nonetheless, deficits for fear and sadness were more strongly correlated with facial affect recognition and vocal affect recognition compared to the other emotions. 
Finally, in a much more extensive review, Brook et al. (2013) examined 58 studies which looked at affect recognition in a variety of areas and found that strong evidence could not be found to support either theory. In a pooled effect size analysis of facial and vocal affect recognition studies of specific emotions, they found that no emotions had a strong effect size with psychopathy. Of note, however, was that fear, sadness, as well as disgust, had the highest effect sizes compared to the other emotions. As the authors noted, this cannot be taken as strong evidence for either theory, however it does suggest a trend towards the view that psychopaths are more impaired in processing the negative emotions of sadness and fear compared to other emotions.

Emotion Production. In comparison to emotion perception in psychopathy research, studies on emotion production are scarce. This is likely due to the difficult nature of eliciting and analyzing emotional responses. Previous studies have mainly used natural and elicited vocal and text samples to explore emotion production (e.g., Louth, Williamson, Alpert, Pouget, \& Hare, 1998; Hancock et al., 2013). Louth et al. (1998) performed the only study on vocal affect in the literature. They looked at 20 offenders, 10 whom were labeled as clinical psychopaths. The authors found that clinical psychopaths failed to differentiate between affective and neutral words with vocal emphasis compared to controls who spoke louder about affective words than neutral words. This suggests that psychopaths are impaired in their production of emotion as well as in processing emotion.

Most studies of emotion production exist in the area of text production (Garcia \& Sikström, 2014; Hancock et al., 2013; Preotiuc-Pietro et al., 2016; Sumner et al., 2012; Wald et al., 2012). The majority were done with non-clinical populations, with only Hancock et al. (2013) investigating an incarcerated clinical sample. Overall, these studies found that non- 
clinical psychopaths produced significantly more negative polarity content compared to controls Garcia \& Sikström, 2014; Preotiuc-Pietro et al., 2016; Sumner et al., 2012; Wald et al., 2012)., with only clinical psychopaths who scored high on Factor 1 producing significantly more negative polarity text compared to their controls (Hancock et al., 2013). These were in contrast to the findings from the studies on emotion processing studies and vocal affect production which suggested that emotions, particularly negative ones, were significantly reduced in both nonclinical and clinical psychopaths. This suggests that the emotional content of language may represent a unique perspective on emotion production in psychopaths.

The studies that analyzed emotion in the text of psychopaths often also looked at other aspects of the text, and therefore overlap entirely with studies on psychopathy prediction with linguistic features. For this reason, studies on emotion production with text will be reviewed in detail in the discussion of linguistic correlates of psychopathy.

Discussion. Overall, numerous studies have found evidence to support the presence of emotion processing deficits in both non-clinical and clinical psychopathy. These studies suggest that emotion may be particularly useful for predicting psychopathy. However, as they are only about emotion processing and not production, it is less clear whether emotion production by psychopaths follows a similar pattern. Fewer studies have been done in emotion production, and they only demonstrate that psychopaths use more negative words than non-psychopaths. In addition, it is unclear whether all emotions are impaired or only specific negative emotions are impaired. However, negative emotions appear more affected than other emotions in psychopaths compared to non-psychopaths.

\section{Text Mining}


Text mining, also known as computational linguistics, provides a method of automatically extracting and analyzing information from text (Stavrianou, Andritsos, \& Nicoloyannis, 2007). It is often used by psychologists to examine associations between language and psychological concepts (Yarkoni, 2010). In particular, information such as the emotional and semantic content of text can be extracted.

Sentiment Analysis. Sentiment analysis (SA) is the area of text mining that focuses on extracting emotion information from text (Liu, 2012). In psychology, SA has been used as a method of analyzing emotion production (e.g., Schwartz et al., 2013). The focus of most SA systems is extracting the polarity of emotion. Polarity can be expressed as a binary (positive or negative) or a ternary (positive, negative, or neutral) expression. Some systems also extract the intensity or arousal of the emotion, which can be conveyed as a categorical scale (e.g., Very Negative to Very Positive) or a continuous scale (e.g., -1 to +1 ) (Liu, 2012). There are two main approaches in SA: Lexicon-based and machine-learning SA. Successful SA systems have been implemented using both of these approaches. The following sections describe the two approaches, including examples of each. First, however, terminology in SA is discussed.

Terminology. In SA research, definitions of emotion terms often differ from the definitions used in psychology. Here, the terms emotion, affect, mood, and sentiment are discussed in relation to both fields. In psychology, researchers have provided many definitions of emotion, however a consensus has not been reached yet (see Barrett, 2006; Izard, 2010; Kleinginna \& Kleinginna, 1981 for reviews). Nonetheless, a common definition of emotion is that it is a brief, intense psychological state that is in response to some event or object (Gooty, Connelly, Griffith, \& Gupta, 2010; Fisher, 2000; Scherer, 2000). In addition, it is generally agreed that valence and intensity are components of emotion (Barrett \& Russell, 1998; Munezero 
et al., 2014). Affect is defined as a general term in psychology which is used to refer to any long lasting emotional psychological states. For example, mood is referred to as a kind of affect since it is long lasting (Gooty et al., 2010). Specifically, mood is defined as a psychological state that is longer lasting and less intense than emotion (Fisher, 2000; Gohm \& Clore, 2002). Finally, in psychology, sentiment is longer lasting than mood. Specifically, it is defined as it is a disposition to have a certain emotional response to an event or object (Cattell, 2006; Gordon, 1981; Kelly \& Barsade, 2001).

It has been noted that emotion terminology is often not well-defined in SA research (Munezero et al., 2014; Balahur, Hermida, \& Montoyo, 2012). One of the only definitions of emotion put forward in SA is that it is a subjective feeling or thought (Liu, 2012). Typically, emotion is described in terms of discrete emotion states, such as Plutchik's (1980) eight basic emotions or Ekman's (1999) six basic emotions (e.g., Aman \& Szpakowicz, 2007; Mohammad \& Turney, 2013; Strapparava \& Mihalcea, 2008). For example, Ekman’s basic emotions are: anger, happiness, surprise, disgust, sadness, and fear. This is in comparison to psychology, where emphasis is on a description of emotion rather than its discrete states. In regard to affect, most SA studies use affect as a general word for anything relating to emotion (e.g., Strapparava \& Valitutti, 2004; Thelwall et al., 2010). Mood has been used to refer to both public and individual long-term feelings about an event, entity or object (Bollen, Mao, \& Pepe, 2011; O’Connor, Balasubramanyan, Routledge, \& Smith, 2010; Thelwall et al., 2010; Yang, Lin, \& Chen, 2007). Public mood is typically analyzed using individual's twitter posts (Bollen et al., 2011; O'Connor et al., 2010). Individual mood has been analyzed using blogs and social networks where users' annotate their posts with their mood (Thelwall et al., 2010; Yang et al., 2007). Finally, the most important difference in definition between psychology and SA is for sentiment. In SA, sentiment 
is generally defined in relation to opinion. An opinion contains a target (e.g., an entity) and a sentiment towards the target. In this case sentiment expresses the polarity of the opinion (e.g., negative or positive) (Liu, 2012; Mohammad \& Turney, 2013; Thelwall et al., 2010).

Lexicon-based Sentiment Analysis. Lexicon-based SA determine sentiment polarity based on a lexicon of words (i.e., sentiment words) and their associated polarities (Ignatow \& Mihalcea, 2016). Words can also be labeled according to discrete emotions (Aman \& Szpakowicz, 2007; Mohammad \& Turney, 2013; Strapparava \& Mihalcea, 2008). Such lexicons can be created manually or semi-automatically. In the manual method, researchers must provide all sentiment words themselves (Liu, 2012). Semi-automatic methods, however, only requires a few words and polarities from the researcher. These initial sentiment words are referred to as seed words or the seed set. A popular semi-automatic method is propagation, also known as bootstrapping. Here the seed set is expanded by finding words that correlate with the seed words in dictionaries or texts (Liu, 2012; Pang \& Lee, 2008).

In addition to a sentiment lexicon, most current lexicon-based systems also use characteristics of the sentence, such as intensifiers (e.g., "very"), negators (e.g., "not"), and sentiment rules (e.g., phrases on either side of "but" have opposite polarities) to determine polarity (Ding, Liu, \& Yu, 2008; Taboada, Brooke, Tofiloski, Voll, \& Stede, 2011). The final polarity of a sentence or phrase is determined using an aggregation function which takes into account polarity information from both the lexicon and characteristics of the sentence (Liu, 2012). Aggregation functions vary from simple to complex computations. For example, in $\mathrm{Hu}$ and Liu (2004), the final polarity of a sentence is determined by whether there are more positive or negative sentiment words in the sentence. Other aggregation functions incorporate more information about the sentence as well as mathematical concepts (e.g., Dey \& Haque, 2009). 
Many examples of sentiment lexicons exist in the literature (e.g., Hatzivassiloglou \& McKeown, 1997; Hu \& Liu, 2004; Kim \& Hovy, 2006; Turney, 2002; Wiebe, Wilson, \& Cardie, 2005; Cambria, Olsher, \& Rajagopal, 2014). For a review of lexicon-based SA and sentiment lexicons, see Pang \& Lee (2008) or Taboada et al. (2011). In this paper, some of the most popular approaches will be discussed: Dictionary of Affective Language (DAL; Whissel, 1989), Affective Norms for English Words (ANEW; Bradley \& Lang, 1999), SentiWordNet (Esuli \& Sebastiani, 2006), and Word-Emotion Association Lexicon (EmoLex; Mohammad \& Turney, 2010).

DAL is a sentiment lexicon consisting of 8,742 English words manually created in the 1980s and later revised (Whissel, 1989). Like ANEW, ratings are provided for each word in terms of three dimensions. Each word is given a rating from one to three based on ternary polarity, arousal, and imagery (i.e., ease of visualization). The lexicon sources its words from a large number of different natural language samples, and therefore is said to have broad coverage (Agarwal, Biadsy, \& Mckeown, 2009). However, because it was created in the 1990s, DAL does not include more recent words and lexical features (e.g., acronyms, slang). Therefore, it performs poorly on modern texts, especially those derived from social media (Hutto, Yardi, \& Gilbert, 2013).

ANEW is a sentiment lexicon containing 1,034 English words which was manually created in the late 1990s. Ratings are provided for each word in terms of polarity, arousal, and dominance (dominated to in control) on a scale from one to nine, with a neutral midpoint at 5 (Bradley \& Lang, 1999; Whissel, 2008). ANEW has a significant advantage over other sentiment lexicons. Due to its numeric rating of polarity, it can describe the intensity or strength of a 
word's polarity. Similar to DAL, however, it lacks certain modern slang words and acronyms (Hutto et al., 2013).

SentiWordNet is a popular lexicon that currently contains 28,431 sentiment words (Esuli \& Sebastiani, 2006; Baccianella, Esuli, \& Sebastiani, 2010). Each word and category in SentiWordNet is given three numeric scores based on positive, negative and neutral polarity. Each score ranges from 0 to 1 . SentiWordNet was built using a seed set of sentiment words and the WordNet dictionary. A classifier was trained to find sentiment words using the seed set and semantic relations in WordNet.

Finally, EmoLex is a manually created lexicon that currently contains 14,182 words (Mohammad \& Turney, 2010; 2013). Words are labeled according to Plutchik's (1980) eight basic emotions: anger, anticipation, disgust, fear, joy, sadness, surprise, and trust. The lexicon was created through internet crowdsourcing, where around 1,000 participants annotated a provided dictionary with emotion labels. Each word was annotated by five different people and the emotion that the majority agreed on was chosen as its label (Mohammad \& Turney, 2010).

Machine Learning Sentiment Analysis. Machine learning SA, also known as statistical SA, uses machine learning to learn which text inputs correspond to which polarity from examples in a training set of documents (i.e., a corpus) (Ignatow \& Mihalcea, 2017). Therefore, the task is viewed as a classification problem. A classifier algorithm is used to create a model which learns the appropriate matches between text inputs and polarity outputs. The resulting trained model is applied to a test corpus it has never been exposed to before and makes classification predictions based on its training (Liu, 2012). 
The majority of machine learning method approaches in SA are supervised, that is, the training corpus used contains sentences which are labeled according to their appropriate polarity, typically by a human rater (Liu, 2012). Corpora have been created for SA containing text from different domains, such as product reviews (Hu \& Liu, 2004), movie reviews (Pang \& Lee, 2005), or MySpace comments (Thelwall et al., 2010). The domain a training corpora comes from is important, as a machine learning SA system trained on one domain cannot be used in another domain without a significant decrease in performance (Aue \& Gamon, 2005).

Examples of machine-learning SA systems include: Stanford Deeply Moving SA system (Socher et al., 2013), and the SentiStrength system (Thelwall et al., 2010). Stanford's sentiment system, Deeply Moving, is based on a deep learning network method referred to as Recursive Neural Tensor Network. A deep learning network is a type of neural network, a network of nodes connected via weights, which contains many layers of processing (Schmidhuber, 2015).

Normally a neural network contains an input and output layer, and sometimes a few processing layers in between (Feldman \& Sanger, 2007). Socher et al. (2013) parsed the Pang and Lee (2005) movie review corpus into syntactic structures of 215,154 unique phrases. These phrases were labeled for sentiment at each level of the syntactic structure (e.g., phrases of various sizes, individual words) on a 7-point scale from very negative to very positive. This provides a corpus labeled with fine-grained sentiment for the system. The supervised classifier is trained using semantic representations of the syntactic structures of the corpora created by the neural network as well as the associated sentiments. The method provides a high level of performance due to its representation of the sentence at various structural levels as well as its ability to take word order into consideration (Liu, 2015; Ribeiro et al., 2016). 
The SentiStrength system is a hybrid system which uses both lexicon and machine learning methods. The sentiment lexicon was made for the system and contains 763 words. Words are described in terms numeric polarity from 2 to 5 for positive words and -2 to -5 for negative words. In SentiStrength, sentences are initially labelled according to the sentiment lexicon. These initial ratings are then optimized using a machine learning algorithm trained on a MySpace comment corpora. The algorithm works by determining whether increasing or decreasing a word's strength by 1 point increases classification accuracy by 2 units. If it does, then that single unit increase or decrease of word strength is maintained and becomes the word's new sentiment strength for that text. Afterwards, the sentence is processed using various sentence characteristics such as intensifiers, negators, and emoticons. The final scores of a sentence is given by both the most positive and the most negative word in the sentence. Therefore, each sentence has a positive and negative score to demonstrate the possible mixed nature of sentiment in that sentence (Thelwall et al., 2010).

Discussion. Lexicon-based and machine learning approaches both have benefits and limitations associated with them. Lexicon-based approaches are useful as they can often be used as they are and do not require supplementary material or preparation. However, a drawback of lexicon-based systems is that the words and associated sentiment values in the lexicon are fixed (Liu, 2012). For example, lexicons made at an earlier time, such as DAL and ANEW do not have many modern terms and slang (Hutto et al., 2013). Therefore, these lexicons perform poorly on modern texts, especially those derived from social media (Hutto, Yardi, \& Gilbert, 2013). However, even more modern lexicons like SentiWordNet and EmoLex have the limitation of being too fixed. Sentiment lexicons, in general, cannot adapt their sentiment values according to domains or sentences where words may be used differently. For example, the verb "love" is used 
more casually in social media compared to normal text (Thelwall et al., 2010). A sentence sometimes may also not convey sentiment despite containing a sentiment word. For example, "I am looking for a good car to buy" contains the sentiment word "good", but as a sentence does not express a positive or negative sentiment. Despite these drawbacks, lexicon-based approaches are still more popular than machine learning approaches in the psychology literature simply due to their practicality (Schwartz et al., 2013).

Machine learning systems are known to be able to reach higher performance standards than lexicon-based systems (Chaovalit \& Zhou, 2005; Kennedy \& Inkpen, 2006). However, these systems are domain-dependent, and when systems trained on one domain are used on data from another domain, performance drops dramatically (Aue \& Gamon, 2005). In addition, not many corpora exist which are well-annotated and large enough for sufficient training of classifiers (Agrawal \& An, 2012). Therefore, researchers must often create their own corpora, which can be very effortful and time consuming (Liu, 2012).

Semantic Analysis. Many methods in text mining exist to extract semantic information. Semantic analysis can be divided into two broad approaches according to Schwartz et al. (2013): closed-vocabulary analysis, which is based on a lexicon, and open-vocabulary analysis, which is not based on a lexicon.

Closed-vocabulary analysis. Closed-vocabulary analysis uses pre-defined categories of words to determine the semantic content of text. Lexicons describe a variety of features, including sentiment, semantic, and grammatical categories. They are typically paired with a function to determine the semantic content of text. This function may be a simple word count or may take into account the surrounding sentence structure of words. 
Examples of popular closed-vocabulary methods in psychology include General Inquirer lexicon (GI; Stone, Dunphy, \& Smith, 1966), Linguistic Inquiry and Word Count (LIWC; Pennebaker et al., 2007), Wmatrix (Rayson, 2008), and Sentiment Analysis and Social Cognition Engine (SÉANCE; Crossley et al., 2017).

The GI lexicon is a manually created lexicon. The lexicon was first made in the 1960's, but words are continually added to it (Stone et al., 1966). Currently, the lexicon contains 11,788 words organized into 182 categories related to semantic and sentiment concepts. These categories include 63 word lists from the Laswell dictionary (Lasswell \& Namenwirth, 1969).

The LIWC system is built on a manually-created lexicon of 4,500 words organized into 80 semantic and sentiment categories (Pennebaker et al., 2007). The system is very popular in behavioural research due to its inclusion of cognitive concepts. The LIWC system determines semantic content using a simple word count method by first determining if a word belongs to a certain category and then counting how often that category appears in the text (Pennebaker et al., 2007).

Wmatrix is a text analysis tool that compares texts based on semantic and sentiment information it extracts from the texts (Rayson, 2008). Words are annotated according to 21 broad semantic classes (e.g., money, time, social actions), each with sub-categories, making a total of 232 sub-categories. Wmatrix takes into account information such as word frequency and surrounding context when determining syntactic and semantic similarity between texts (Rayson, 2008).

Finally, SEANCE is a recent linguistic analysis system which combines several preestablished lexicons to make them easily accessible (Crossley et al., 2017). Lexicons included 
are: the Laswell dictionary (Lasswell \& Namenwirth, 1969) which contains nine semantic categories; the Geneva Affect Label Coder (GALC; Scherer, 2005) which has 36 specific emotion categories and two polarity categories; EmoLex (Mohammad \& Turney, 2013) which contains eight specific emotion categories and two polarity categories; SenticNet (Cambria et al., 2012) which is an extension of WordNet that describes words according to sensitivity, aptitude, attention, pleasantness scales; and finally, GI and ANEW which have already been described. The SEANCE function takes into account the grammatical category of the word in determining semantic and sentiment category. In addition, the tool includes 20 sentiment and semantic component categories which are made by combining the scores of categories from the other lexicons.

Open-Vocabulary Semantic Analysis. Open-vocabulary analysis uses statistical methods to automatically extract semantic information. Rather than being based on pre-existing manmade categories, open-vocabulary analysis extracts information based on the text provided (Schwartz et al., 2013). Therefore, it is able to capture information that may not have been represented in a pre-made category.

Examples of open-vocabulary analysis include: the bag-of-words (BOW) model, and Latent Semantic Analysis (LSA; Landauer \& Dumais, 1997). BOW models are the most common and simple type of open-vocabulary analysis. In this model, all words in a text are used to represent the text in a vector space model. The word vectors can be weighted according to different methods. In binary weighting, the weight simply reflects whether the word is present or not. In frequency weighting, the weight reflects how often the word appears given the total number of words in the text. Finally, term frequency-inverse document frequency (TF-IDF) 
weighting reflects how often the word appears given how often it appears in the corpora as a whole (Feldman \& Sanger, 2007).

LSA is based on the same vector space model theory of BOW. However, rather than representing the text with words or frequencies, LSA uses abstract or latent concepts derived from the text to represent the text (Landauer \& Dumais, 1997). Therefore, it is able to represent the text at a higher conceptual level than the BOW model and capture associations between words (Deerwester, Dumais, \& Harshman, 1990).

Discussion. Semantic representation of text can either be through categorical closedvocabulary analysis or statistical open-vocabulary analysis. Closed-vocabulary analysis is restricted as it contains a fixed list of words and categories. This is a problem as words may be polysemous and belong to more than one semantic category. This can lead to miscategorising words. In addition, like sentiment lexicons, the fixed nature of semantic lexicons means that lexicons created at an earlier time do not include modern words. The main problem with both sentiment and semantic lexicons is that they are restricted to the lists that exist. Lexicons must be very large in order to account for all the words encountered in a text. Open-vocabulary analysis does not face this problem as they represent the text using its own words. However, most openvocabulary methods do not take into account the possible information provided by sentence structure, while some closed-vocabulary methods do. In addition, while the information provided by open-vocabulary analysis may represent the text as a whole more accurately, closedvocabulary analysis is more useful for psychologists studying specific categories. For this reason, most behavioural studies have used closed-vocabulary methods in their research (e.g., Hancock et al., 2013; Oberlander \& Nowson, 2006).

\section{Linguistic Correlates of Psychopathy}


As mentioned previously, the relation between personality and language suggests a relation between personality disorders and language, specifically psychopathy. Studies which have previously explored this used primarily SA and semantic tools to analyze text from psychopaths (Garcia \& Sikström, 2014; Hancock et al., 2013; Preotiuc-Pietro et al., 2016; Sumner et al., 2012; Wald et al., 2012).

Non-clinical Studies. The majority of studies on language and psychopathy have been done with non-clinical populations. These studies mostly used un-elicited, natural language text from social media sources as their data. In addition, a variety of non-clinical psychopathy measures were used to determine psychopathy in these samples.

Garcia and Sikström (2014) examined correlations between non-clinical psychopathy and language used in the Facebook posts of 304 users. They measured non-clinical psychopathy using the psychoticism scale from the EPQR-S, a personality trait questionnaire. The psychoticism scale has been previously related to non-clinical psychopathy (Zuckerman, 1991). They analyzed the language used in Facebook status updates by creating a semantic representation of the posts using LSA, therefore achieving a broad understanding of the topics individuals posted about. In addition, they measured the semantic polarity of these semantic representations using the ANEW sentiment lexicon, and also measured the 'prototypicality' of the posts (i.e. how much they veered from average content). Garcia and Sikström found negative correlations between non-clinical psychopathy and positive polarity as well as prototypicality, suggesting non-clinical psychopaths used more negative polarity words and unusual language. However, in this study, the strongest correlation was found between non-clinical psychopathy and the overall semantic representation of the posts. 
Sumner et al. (2012) used the more semantically detailed LIWC2007 to analyze language from 2,927 users in their Twitter posts. In addition, non-clinical psychopathy was measured using the Short Dark Triad. The Big Five Personality traits were also measured using the Ten Item Personality Inventory (TIPI) (Gosling, Rentfrow, \& Swann, 2003). Tweets were analyzed using the categories from LIWC. They found that non-clinical psychopathy scores were significantly correlated with the use of swear words, anger, death, as well as negative emotion. Non-clinical psychopathy scores were also negatively correlated with positive emotion. In terms of semantic content, psychopathy scores were moderately correlated with body and sexual words as well as strongly and negatively correlated with family words. Using all of the LIWC categories as well as additional features related to the users' twitter usage (e.g., number of followers), psychopathy was able to be predicted with a modest level of accuracy. It should be noted that LIWC categories were more strongly correlated with non-clinical psychopathy than user profile information and therefore, LIWC likely contributed more to the prediction of nonclinical psychopathy. Wald et al. (2012) used the same data, but only looked at data from 2,916 participants. Similarly, they were able to predict psychopathy using linguistic features, including LIWC. However, they did not compare prediction models to explore which predicted psychopathy best.

Recently, Preotiuc-Pietro et al. (2016) did an even more thorough analysis of by looking at the predictive effects of various linguistic measurements for non-clinical psychopathy. Specifically, they processed Twitter posts from 419 users who were measured on non-clinical psychopathy using the Dirty Dozen Questionnaire (Jonason \& Webster, 2010). Like Sumner et al., (2012), Preotiuc-Pietro et al. used the LIWC 2007. However, they also processed the posts by extracting unigrams, emotion words from the EmoLex emotion lexicon, and word clusters (i.e. 
topics). Controlling for gender and age, they found strong, significant correlations between psychopathy and the death, anger, and negative polarity and body categories of LIWC. In addition, from the EmoLex lexicon the strongest correlations with psychopathy were negative polarity, disgust, fear, anger, and sadness. Finally, for word clusters, the highest correlations with psychopathy were for criminal activity and names. Overall, using a linear regression, PreotiucPietro et al. found that all linguistic features strongly predicted non-clinical psychopathy, with LIWC being the best predictor and Word Clusters and EmoLex following closely behind. Unigrams were not as strong in predicting non-clinical psychopathy compared to the other linguistic features. Their results support the view that negative polarity may be a robust predictor of non-clinical psychopathy, particularly as the relevant categories of LIWC and the word clusters were all related to negative polarity, with the exception of the body and names category.

Clinical Studies. The above studies suggest a strong relation between non-clinical psychopathy and sentiment polarity, as well as non-clinical psychopathy and certain semantic categories (e.g., body words). However, only one study has explored language production in clinical psychopathy. Hancock et al. (2013) looked at the detailed semantic and sentiment content of the homicide narratives produced by psychopathic and non-psychopathic offenders. Participants were measured on clinical psychopathy using the PCL-R. Their narratives were analyzed using Wmatrix (Rayson, 2008) which extracts semantic categories from text similar to LIWC. As well, they used the DAL sentiment lexicon. Therefore, they were able to measure the sentiment polarity as well as polarity strength of the narratives. However, as opposed to the earlier findings with non-clinical psychopaths, overall PCL-R score was not significantly correlated with negative emotion. Instead, only Factor 1 of the PCL-R was significantly correlated with negative emotion words. In addition, only Factor 1 of the PCL-R was correlated 
with decreased polarity intensity. The semantic content, however, was in agreement with the non-clinical psychopathy studies. Hancock et al. found that overall PCL-R score was significantly correlated with the Wmatrix categories of food, drink, clothing and money and negatively correlated with the categories of family and religion.

Discussion. Although only a few studies have looked at psychopathy and language production, there appears to be trend towards a relationship between linguistic features and psychopathy, particularly sentiment and semantic features. All studies on non-clinical psychopathy found a relation between negative polarity and high psychopathy scores. The only study that did not find this relation was Hancock et al. (2013) who evaluated individuals with clinical levels of psychopathy. Therefore, it is uncertain whether this relation between sentiment and psychopathy exists in clinical populations as well.

In addition, psychopathy scores were associated with certain semantic categories. Many of these categories were previously suggested as being associated to Maslow's hierarchy of needs by Hancock et al., (2013). Maslow's hierarchy of needs is a theory of motivation which states that there are different levels of needs (Maslow, 1954). According to Maslow, low level physical needs (e.g., body safety, food) must be satisfied first before trying to satisfy high level social needs (e.g., family, friends, religion). In addition, if low level needs are not satisfied, then the individual is more likely to experience poor psychological health. Hancock et al. (2013) theorized that psychopaths focus on physical needs and put less focus on social needs. This was found to be reflected in the literature. For example, psychopathy was associated with body words in non-clinical participants (Preotiuc-Pietro et al., 2016; Sumner et al., 2012). Psychopathy was also negatively correlated with family words in clinical (Hancock et al., 2013) and non-clinical psychopathy (Sumner et al., 2012). Additionally, Hancock et al. found associations between 
clinical psychopathy scores and food, drink, clothing and money words (i.e. physical needs) and a negative correlation between psychopathy and religion. Finally, Sumner et al. (2012) found a relation between non-clinical psychopathy and sexual words. Overall, it appears that in both clinical and non-clinical populations, psychopathy is related to using more words about physical needs and fewer words about social needs.

Finally, as mentioned, even among the non-clinical studies, a variety of methods were used, in terms of both SA classification and non-clinical psychopathy measurement. Although all studies used lexicon-based methods, different lexicons were used, some of which held more detailed information than others. For example, only Hancock et al. (2013), who utilized DAL, described the polarity strength of texts and found a relation between factor 1 psychopathy scores and decreased polarity strength. Although Garcia and Sikström (2014) used a lexicon with sentiment strength marked as well, they did not explore this feature. Since Hancock et al. also did not find a relation between overall clinical psychopathy scores and polarity, despite this finding being consistent with non-clinical psychopathy, it may be the case that overall nonclinical psychopathy is associated with decreased sentiment strength. Related to this is the fact that none of the non-clinical studies used psychopathy measures which had a factor structure. Therefore, it cannot be determined whether the relations Hancock et al. found between factor 1 psychopathy scores and polarity as well as polarity strength are also present in non-clinical populations.

\section{Current Study}

The current study analyzed text from clinical and non-clinical psychopaths to determine which linguistic features are associated with psychopathy. Text collected from individuals who 
score high on psychopathy were analyzed in terms of sentiment and semantic content. The research questions explored in this study are:

1. Is there a relationship between sentiment polarity and overall psychopathy score for clinical and non-clinical psychopathy?

2. Is there a relationship between sentiment strength and psychopathy score for clinical and non-clinical psychopathy?

3. Is there a relationship between sentiment polarity and Factor 1 of psychopathy for clinical and non-clinical psychopathy?

4. Is there a relationship between psychopathy score and Maslow's hierarchy of needs for both clinical and non-clinical psychopathy?

Based on previous studies, the following hypotheses were proposed. Studies on non-clinical psychopathy suggest that a relationship exists between psychopathy and negative sentiment. In addition, the strong evidence for emotion perception impairments in both clinical and nonclinical psychopathy suggests that emotion production impairments exist for both samples as well. Therefore, I expected that overall psychopathy would be related to negative sentiment in both clinical and non-clinical psychopathy. Given psychopathy is associated with significantly decreased emotion perception, I hypothesized that high psychopathy scores would be correlated with lower sentiment strength in language production for both non-criminal and criminal groups. Following the theory that clinical and non-clinical psychopaths express the same impairments to different degrees, I also expected that psychopathy scores in the clinical group would be associated with lower sentiment strength than in the non-clinical group. Due to the findings from Hancock et al. (2013), as well as the relationship between emotion and Factor 1 of psychopathy (Hare \& Neumann, 2008), I also expected that a relation would be found between sentiment 
polarity and Factor 1 score for both clinical and non-clinical psychopathy. Finally, given findings on semantic content and psychopathy, I hypothesized that psychopathy would be correlated with words related to physical needs (i.e., body, food) and negatively correlated with words related to social needs (i.e., family, religion) for both clinical and non-clinical psychopaths.

\section{Method}

\section{Participants}

The current study analyzes transcribed interviews from both clinical and non-clinical samples. Data from the clinical participants came from a previous study (Forth \& Flight, 2007), while data from non-clinical participants was collected specifically for this study.

Non-Clinical Sample. Non-clinical data was collected from 45 male participants studying at Carleton University in Ottawa, Canada. Males were chosen in order to match the clinical sample I had access to which was all-male. The ages of participants ranged from 18 to 39 years old. Their ethnicities were: Caucasian (23; 51.11\%), Middle-Eastern (7; 15.56\%), African (11.11\%), South-Asian $(5 ; 11.11 \%)$, and Other $(3 ; 6.67 \%)$. The majority of participants were undergraduates (84.44\%), with a few Master's (13.33\%) and Ph.D. students $(2.22 \%)$ in the sample as well. Participants were recruited through SONA, the university's online study sign-up system, as well as through posters placed around the university. Participants recruited through SONA were compensated with $1 \%$ credit toward their course. Participants recruited through posters were compensated with a \$10 Tim Horton's gift card.

Clinical Sample. Clinical data came from 25 male juvenile offenders incarcerated at Bluewater Youth Centre in Goderich, Ontario. Participants' ages ranged from 16 to 18 years old. In addition, the highest grade they completed ranged from Grade 8 to Grade 11, with the 
majority only completing Grade 8 (36\%). Their ethnicities were: Caucasian (68\%), AfricanCanadian (16\%), Aboriginal (8\%), and Other (8\%).

\section{Procedure}

Non-clinical Sample. Participants in the non-clinical sample were interviewed using questions based on items from the PCL youth version (PCL-YV ; Forth \& Kosson, 2003). However, questions about crime were removed and replaced with the participants' opinions about criminal behaviour (e.g., "What personal motives do you think usually causes someone to commit a crime?"). The full set of questions used can be found in Appendix A. It should be noted that the interview used a semi-structured format so many questions were made based on the participant's response. Audio from these interviews were transcribed to create the text analyzed in this study.

After completing the interview, participants were asked to fill out the Self-Report Psychopathy Scale - Short From (SRP-III-SF; Paulhus, Neumann, \& Hare, in press), which measures non-clinical psychopathy (Appendix B). The SRP-III-SF consists of 29 items which are rated on a scale of one (Disagree Strongly) to five (Agree Strongly) by the participants themselves. The scale has previously been shown to correlate strongly with the PCL-R, as well have strong reliability and validity (Dotterer et al., 2016). Little work has been done in exploring the validity of SRP-III-SF in cultural contexts beyond North America (Gordts, Uzieblo, Neumann, Van den Bussche, \& Rossi, 2015). However, as the majority of participants in the non-clinical sample were Caucasian and North American, cultural bias likely did not affect the results. The SRP-III-SF also has items that correspond to the two factors of the PCL-R scale (Gordts et al., 2015). Based on participants' scores and the SRP-III-SF scoring system, only three participants (0.07\%) classified as non-clinical psychopaths. 
Clinical Sample. For the clinical sample, participants were interviewed using questions from the PCL-YV scale (Forth \& Kosson, 2003). The audio from these interviews was then transcribed. To decrease dissimilarity between the clinical and non-clinical groups, however, questions about the clinical participants' crimes were removed from the clinical sample transcripts.

Clinical psychopathy was measured using the PCL-YV. The scale has the same 20 items as the PCL-R, but the descriptions of items and scoring criteria are modified to be suitable for adolescents. The validity of the PCL-YV has been demonstrated in various studies (e.g., Campbell, Porter, \& Santor, 2004; Catchpole \& Gretton, 2003; Neumann, Kosson, Forth, \& Hare, 2006). These studies had samples which were mostly Caucasian. Again, however, the majority of participants in the clinical sample were Caucasian and North American and thus cultural bias was likely not a factor. The PCL-YV can also be broken down into Factor 1 and Factor 2 of psycopathy, similar to the PCL-R. Finally, out of a total of 25 participants, only four were classified as clinical psychopaths according to the PCL-YV scale (16\%).

\section{Text Mining}

Pre-Processing. Text was pre-processed using the Natural Language Processing Toolkit (NLTK; Bird, Loper, \& Klein, 2009). First the researcher's questions and comments were removed. Then the text was converted to lowercase to allow for easier analysis. Words were also stemmed using the Porter Stemmer ${ }^{1}$ so that they were represented in their root forms without prefixes or suffixes (e.g., "run”, “book”). Words that appear frequently (i.e., stop words) were

\footnotetext{
${ }^{1} \mathrm{http}: / /$ tartarus.org/ martin/PorterStemmer/
} 
removed using the stop word list provided in NLTK (e.g., "the”, "of"). In addition, swear words were removed using a swear word list $^{2}$. Finally, all punctuation was removed using NLTK.

Sentiment and Semantic Analysis. Both sentiment and semantic analysis was performed using the closed-vocabulary method, SEANCE (Crossley et al., 2017). Closedvocabulary was chosen as the current study is exploring specific sentiment and semantic categories rather than a broad semantic representation of the text. SEANCE was chosen because it contains several sentiment and semantic lexicons to use for analysis. In addition, it is an opensource tool and is easy to apply. For both sentiment and semantic analysis, the GI lexicon was chosen out of the lexicons available. While other lexicons were available through SEANCE, many do not perform strongly on small text samples (e.g., GALC and Laswell lexicons). GI is also beneficial because it is one of the largest semantic lexicons (Esuli \& Sebastiani, 2007). SEANCE only includes 119 categories of the GI lexicon, since the system includes the Laswell dictionary separately. The system outputs the number of words in a category given the total number of words in the document (i.e., relative frequency) (Crossley et al., 2017).

To analyze sentiment, negative and positive polarity categories from GI were extracted. However, GI does not include sentiment intensity ratings, therefore the arousal category from ANEW was chosen to represent sentiment intensity. To analyze Maslow's hierarchy of needs, the GI categories for Body, Food, Social (friends and family), and Religion were chosen.

\section{Results}

\footnotetext{
${ }^{2}$ http://svn.symfony-project.com/plugins/swFormExtraPlugin/branches/sf1.3/data/badwords_en.txt
} 
Analyses of the data were completed for both clinical and non-clinical samples. For both samples, the distribution characteristics of the variables were analyzed followed by correlational analyses comparing psychopathy measures and semantic features.

\section{Non-Clinical Sample}

Descriptive Analysis. Distribution characteristicss of the non-clinical psychopathy measures and semantic features are shown in Table 1. Shapiro-Wilk tests were used to determine normality for each variable. All psychopathy measures had normal distributions. In addition, all semantic features had normal distributions except for Food, Social, and Religion words.

Boxplots were used to determine the spread of data for both psychopathy scores and semantic features as well as univariate outliers (Figure 1). One univariate outlier was found for Factor 2 (Figure 1a). However, this was not removed as Factor 2 is related to Total SRP and no such outliers were found in Total SRP. Therefore, it is likely that this score is not an error and should not be removed. 13 outliers were found in the semantic feature boxplots for Negative Polarity, Valence, Body, Food, Social, and Religion words (Figure 1b).

To determine possible relationships as well as bivariate outliers between psychopathy scores and semantic features for the non-clinical sample, a scatterplot matrix was created for the data (Figure 2). The matrix contains scatterplots between each variable pair as well as the frequency distribution of each variable along the diagonal of the matrix. The plots of interest are highlighted. These scatterplots suggest that no relationships exist between psychopathy scores and semantic features. If relationships do exist, they are likely to be very small. In addition, the scatterplot matrix was used to determine the presence of bivariate outliers. In the current paper, bivariate outliers are defined as points that are substantially far from other points in a plot. 
Removing univariate outliers would result in the sample size being reduced to 38 participants, while removing bivariate outliers would only decrease the size to 44 . Removing outliers may cause correlation analyses to more accurately reflect the "true" nature of the relationship between variables, but it would also decrease the power of the analyses. The latter is of particular concern in regards to the univariate outliers. Therefore, I decided that I would calculate correlations with univariate and bivariate outliers, without univariate outliers, and finally without bivariate outliers.

Correlational Analysis. Although the scatter matrix suggested no relationships are present between psychopathy scores and semantic features, two-tailed bivariate Pearson correlations were calculated for each pair of these variables. Pearson correlation coefficients and p-values are presented in Table 2. for each psychopathy score and semantic feature pair. Using $\alpha=0.05$, none of the relationships were found to be significant. In addition, due to multiple correlations being performed on the same dependent variables, the alpha value was corrected using Bonferroni adjustment. Since 21 correlations were performed, $\alpha=0.002$.

A scatterplot matrix was then made again for the data with univariate outliers removed (Figure 3). The plots of interest are highlighted. The resulting matrix shows that excluding the outliers removed much of the variability in the relevant plots. In addition, the majority of scatterplots still show a lack of relationship between psychopathy measures and semantic features. However, there seems to be a small positive relationship between Total SRP and Body words, as well as SRP Factor 1 and Body words. There may also be a weak positive relationship between Total SRP and Social words, as well as SRP Factor 2 and Social words. 
Table 1

Mean (M), standard deviation (SD), skew, kurtosis, and Shapiro-Wilk statistic and its p-value for linguistic features and non-clinical psychopathy scores

\begin{tabular}{lcccccc}
\hline Attribute & $M$ & $S D$ & Skew & Kurtosis & $\begin{array}{c}\text { Shapiro-Wilk } \\
\text { Statistic }\end{array}$ & P-value \\
\hline SRP & 51.82 & 12.90 & 0.32 & -0.30 & 0.98 & 0.641 \\
SRP F1 & 15.58 & 4.33 & 0.40 & -0.48 & 0.97 & 0.358 \\
SRP F2 & 10.33 & 2.94 & 0.51 & -0.15 & 0.96 & 0.144 \\
Negative Polarity & 0.15 & 0.02 & 0.71 & 1.00 & 0.96 & 0.080 \\
Positive Polarity & 0.16 & 0.02 & -0.27 & -0.88 & 0.97 & 0.283 \\
Arousal & 0.80 & 0.11 & 0.35 & 0.75 & 0.97 & 0.312 \\
Body & 0.01 & 0.004 & 0.75 & 0.59 & 0.96 & 0.103 \\
Food & 0.005 & 0.002 & 0.85 & 0.85 & 0.95 & 0.039 \\
Social & 0.01 & 0.005 & 0.88 & 1.20 & 0.95 & 0.051 \\
Religion & 0.002 & 0.003 & 2.48 & 7.06 & 0.72 & 0.000 \\
\hline
\end{tabular}

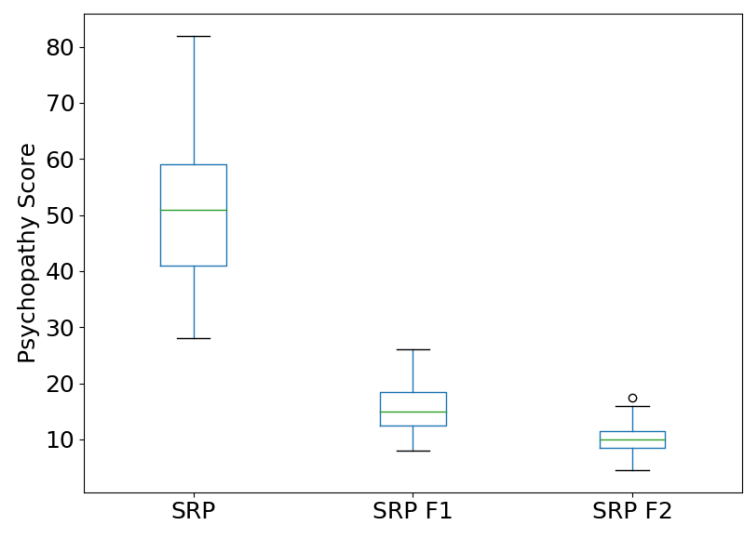

a)

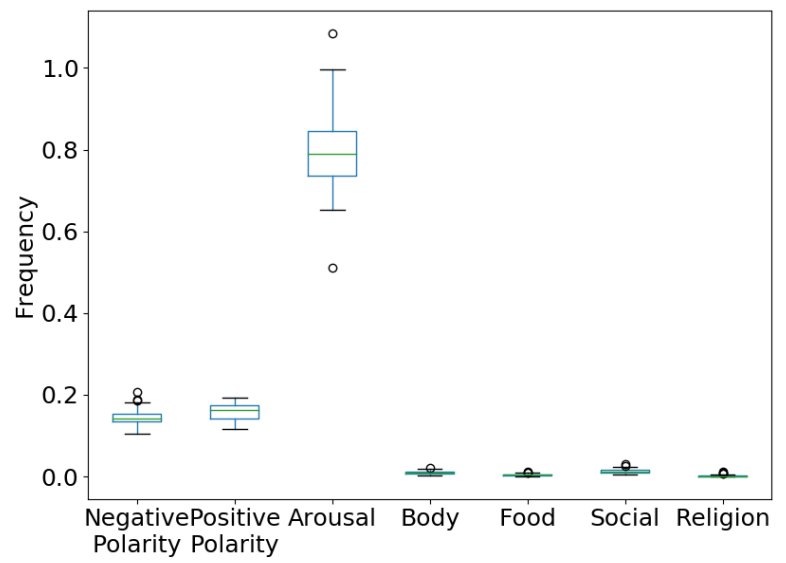

b)

Figure 1. Boxplots for a) psychopathy scales and b) semantic features for non-clinical data with univariate outliers indicated. 


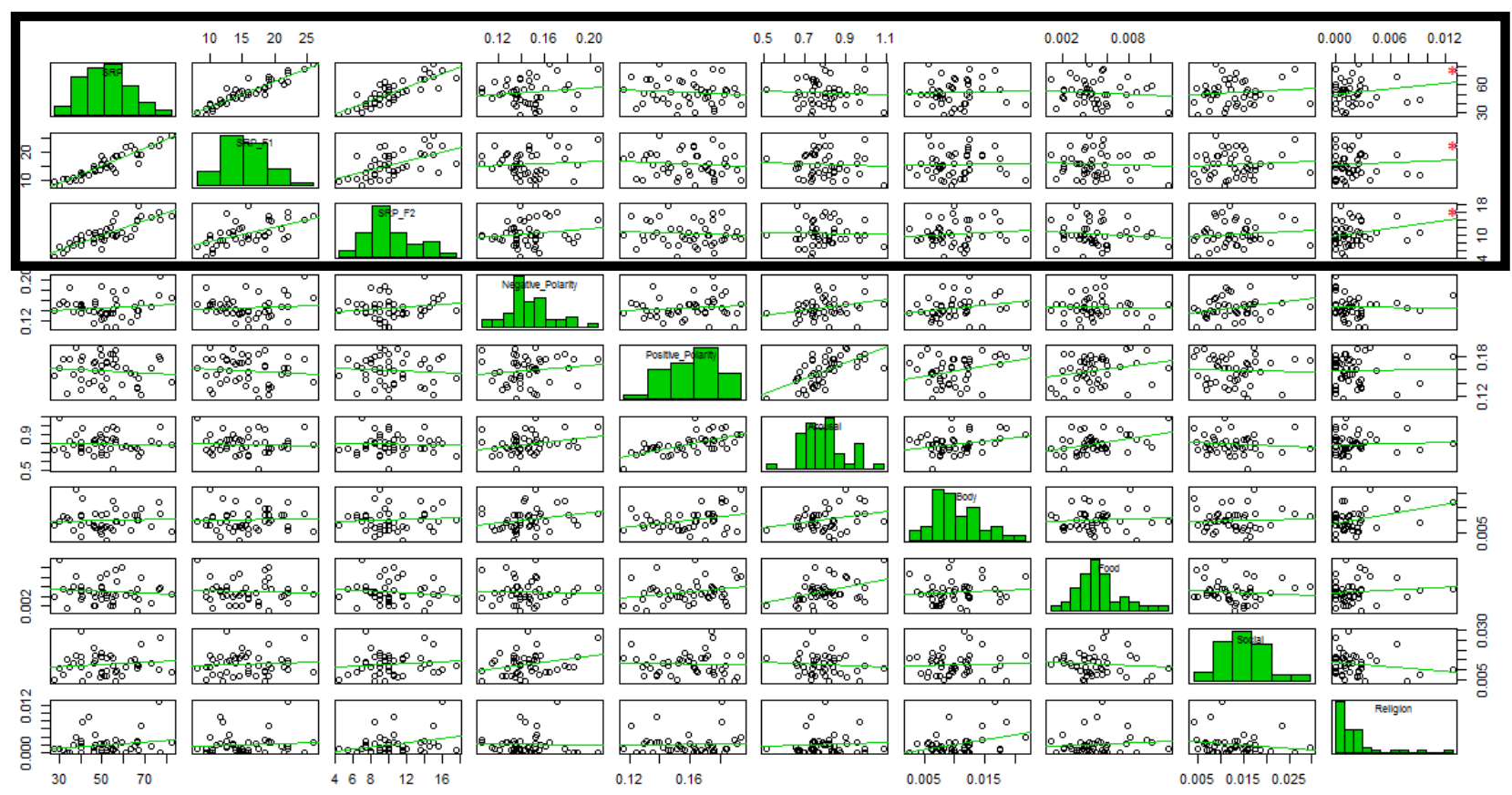

Figure 2. Scatterplot matrix of psychopathy scales and semantic features for non-clinical data with bivariate outliers indicated.

Table 2

Correlation coefficients ( $r$ ) and p-values (p) for two-tailed bivariate Pearson correlation between psychopathy measures and linguistic features for non-clinical data, * = significant at $\alpha=0.05, * *=$ significant at $\alpha=0.002$

\begin{tabular}{lcccccc}
\hline \multirow{2}{*}{ Attributes } & \multicolumn{2}{c}{ Total SRP } & \multicolumn{2}{c}{ SRP Factor 1 } & \multicolumn{2}{c}{ SRP Factor 2 } \\
\cline { 2 - 7 } & $r$ & $p$ & $r$ & $p$ & $r$ & $p$ \\
\hline Negative Polarity & 0.14 & 0.369 & 0.10 & 0.530 & 0.16 & 0.295 \\
& & & & & & \\
Positive Polarity & -0.12 & 0.479 & -0.12 & 0.492 & -0.08 & 0.590 \\
& & & & & & \\
Arousal & -0.05 & 0.757 & -0.06 & 0.698 & -0.02 & 0.915 \\
Body & 0.05 & 0.724 & 0.02 & 0.893 & 0.09 & 0.564 \\
Food & -0.09 & 0.540 & -0.06 & 0.692 & -0.12 & 0.448 \\
Social & 0.11 & 0.467 & 0.09 & 0.554 & 0.11 & 0.469 \\
Religion & 0.19 & 0.210 & 0.09 & 0.581 & 0.29 & 0.050 \\
\hline
\end{tabular}




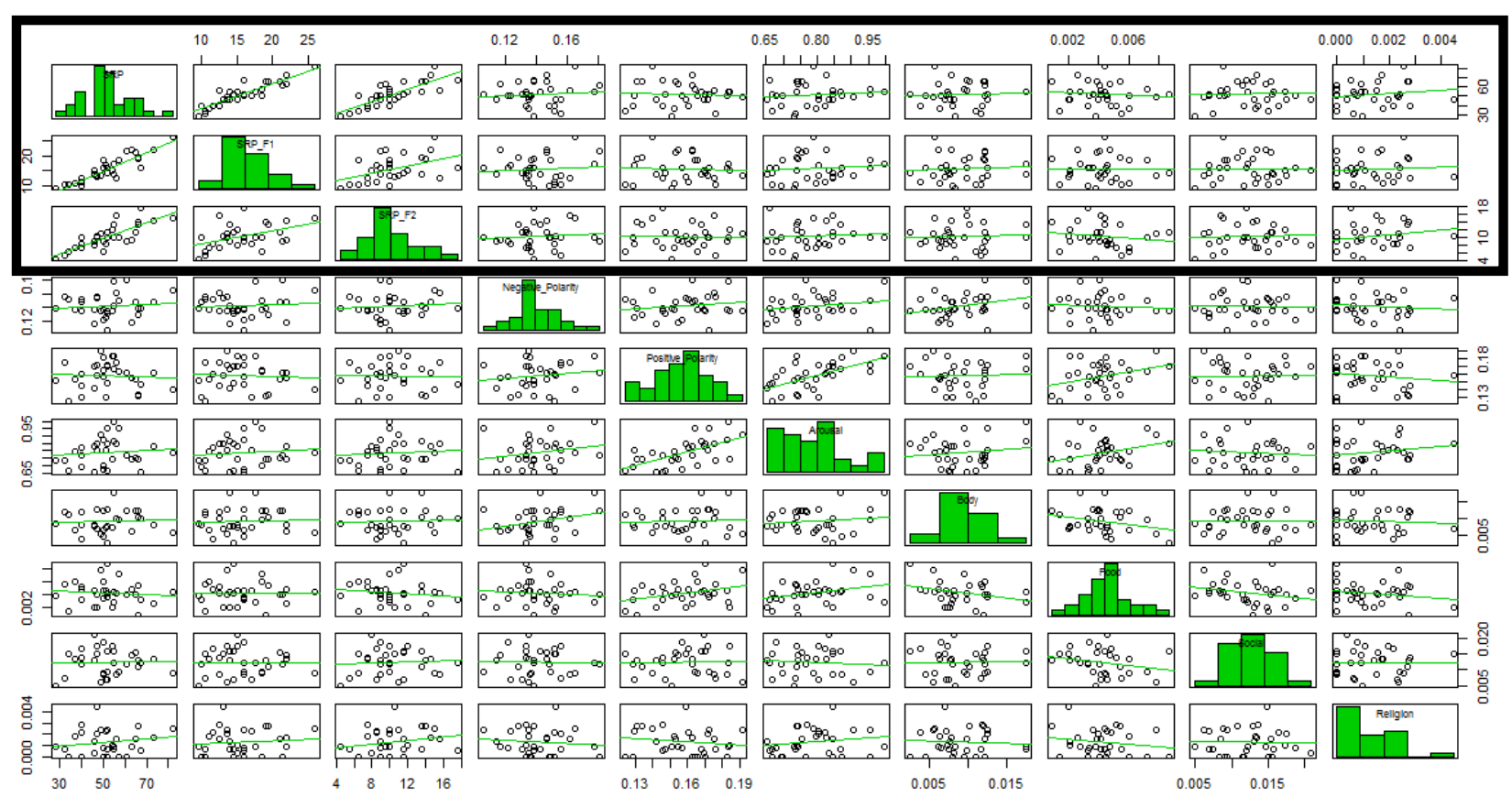

Figure 3. Scatterplot matrix of psychopathy scales and semantic features for non-clinical data without univariate outliers.

Table 3

Correlation coefficients ( $r$ ) and p-values (p) for two-tailed bivariate Pearson correlation between psychopathy measures and linguistic features for non-clinical data excluding univariate outliers, * = significant at $\alpha=0.05, * *=$ significant at $\alpha=0.002$

\begin{tabular}{lcccccc}
\hline & \multicolumn{2}{c}{ Total SRP } & \multicolumn{2}{c}{ SRP Factor 1 } & \multicolumn{2}{c}{ SRP Factor 2 } \\
\cline { 2 - 7 } Attributes & $r$ & $p$ & $r$ & $p$ & $r$ & $p$ \\
\hline $\begin{array}{l}\text { Negative } \\
\text { Polarity }\end{array}$ & -0.07 & 0.684 & -0.12 & 0.524 & 0.02 & 0.933 \\
$\begin{array}{l}\text { Positive } \\
\text { Polarity }\end{array}$ & -0.13 & 0.472 & -0.16 & 0.390 & -0.04 & 0.811 \\
Arousal & 0.11 & 0.543 & 0.10 & 0.578 & 0.09 & 0.639 \\
Body & -0.09 & 0.609 & -0.11 & 0.549 & -0.03 & 0.852 \\
Food & -0.16 & 0.377 & -0.10 & 0.583 & -0.19 & 0.300 \\
Social & 0.02 & 0.896 & -0.01 & 0.970 & 0.06 & 0.756 \\
Religion & 0.04 & 0.826 & -0.03 & 0.852 & 0.13 & 0.461 \\
\hline
\end{tabular}


Table 4

Correlation coefficients ( $r$ ) and p-values (p) for two-tailed bivariate Pearson correlation between psychopathy measures and linguistic features for non-clinical data excluding bivariate outliers, * = significant at $\alpha=0.05, * *=$ significant at $\alpha=0.002$

\begin{tabular}{lcccccc}
\hline & \multicolumn{2}{c}{ Total SRP } & \multicolumn{2}{c}{ SRP Factor 1 } & \multicolumn{2}{c}{ SRP Factor 2 } \\
\cline { 2 - 7 } Attributes & $r$ & $p$ & $r$ & $p$ & $r$ & $p$ \\
\hline $\begin{array}{l}\text { Negative } \\
\text { Polarity }\end{array}$ & 0.09 & 0.562 & 0.06 & 0.714 & 0.11 & 0.468 \\
$\begin{array}{l}\text { Positive } \\
\text { Polarity }\end{array}$ & -0.16 & 0.286 & -0.15 & 0.337 & -0.14 & 0.368 \\
Arousal & -0.05 & 0.745 & -0.06 & 0.690 & -0.02 & 0.907 \\
Body & -0.02 & 0.906 & -0.04 & 0.808 & 0.02 & 0.916 \\
Food & -0.11 & 0.474 & -0.07 & 0.640 & -0.14 & 0.383 \\
Social & 0.14 & 0.372 & 0.11 & 0.477 & 0.14 & 0.370 \\
Religion & 0.01 & 0.932 & -0.08 & 0.617 & 0.15 & 0.345 \\
\hline
\end{tabular}

Table 5

Correlation coefficients ( $r$ ) and p-values (p) for two-tailed bivariate Pearson correlation between psychopathy measures and linguistic features for non-clinical data with swear words included, $*=$ significant at $\alpha=0.05, * *=$ significant at $\alpha=0.002$

\begin{tabular}{lcccccc}
\hline & \multicolumn{2}{c}{ Total SRP } & \multicolumn{2}{c}{ SRP Factor 1 } & \multicolumn{2}{c}{ SRP Factor 2 } \\
\cline { 2 - 7 } Attributes & $r$ & $p$ & $r$ & $p$ & $r$ & $p$ \\
\hline $\begin{array}{l}\text { Negative } \\
\text { Polarity }\end{array}$ & 0.02 & 0.906 & -0.06 & 0.694 & 0.13 & 0.399 \\
Positive & & & & & & \\
Polarity & 0.24 & 0.120 & 0.23 & 0.121 & 0.17 & 0.263 \\
Arousal & 0.01 & 0.965 & -0.08 & 0.613 & 0.13 & 0.399 \\
Body & 0.18 & 0.226 & 0.10 & 0.505 & 0.25 & 0.092 \\
Food & -0.10 & 0.520 & -0.17 & 0.260 & 0.04 & 0.810 \\
Social & 0.03 & 0.827 & -0.05 & 0.737 & 0.15 & 0.328 \\
Religion & 0.18 & 0.245 & 0.10 & 0.506 & 0.24 & 0.115 \\
\hline
\end{tabular}


Table 6

Correlation coefficients ( $r$ ) and p-values (p) for two-tailed bivariate Pearson correlation between psychopathy measures and linguistic features for non-clinical data using LIWC, * = significant at $\alpha=0.05, * *=$ significant at $\alpha=0.002$

\begin{tabular}{lcccccc}
\hline & \multicolumn{2}{c}{ Total PCL-YV } & \multicolumn{2}{c}{ PCL-YV Factor 1 } & \multicolumn{2}{c}{ PCL-YV Factor 2 } \\
\cline { 2 - 7 } Attributes & $r$ & $p$ & $r$ & $P$ & $r$ & $p$ \\
\hline $\begin{array}{l}\text { Negative } \\
\text { Polarity }\end{array}$ & -0.21 & 0.166 & -0.22 & 0.141 & -0.13 & 0.386 \\
Positive & & & & & & \\
Polarity & -0.10 & 0.508 & 0.02 & 0.913 & -0.25 & 0.102 \\
Body & & & & & & \\
Food & -0.23 & 0.137 & -0.26 & 0.081 & -0.11 & 0.489 \\
Family & 0.12 & 0.447 & 0.09 & 0.560 & 0.12 & 0.420 \\
Friend & 0.10 & 0.514 & 0.06 & 0.700 & 0.13 & 0.386 \\
Religion & -0.10 & 0.525 & -0.18 & 0.226 & 0.06 & 0.706 \\
\hline
\end{tabular}


Correlations were then calculated for the data without univariate outliers. Pearson correlation coefficients and p-values are presented in Table 3. for each psychopathy score and semantic feature pair. Similar to the previous analysis, no relationships were found to be significant using either $\alpha=0.05$ or $\alpha=0.002$. In addition, correlations were calculated for the nonclinical data without bivariate outliers. Pearson correlation coefficients and p-values are presented in Table 4 for each psychopathy score and semantic features pair. As in the previous two correlational analyses, no relationships were found to be significant with either $\alpha=0.05$ or $\alpha=0.002$.

Correlations were also performed with swear words included in the text. Two-tailed bivariate Pearson correlations were calculated for each psychopathy measure and each semantic feature for the non-clinical data with swear words included. The results are presented in Table 5. Once again no correlations were found significant with either $\alpha=0.05$ or $\alpha=0.002$..

Finally, in order to determine if the lexicon had an effect on the analysis, semantic categories were also extracted using LIWC2015 (Pennebaker, Boyd, Jordan, \& Blackburn, 2015). However, as LIWC2015 had no category for arousal, this was not included. In addition, the categories Family and Friend were separated instead of being put into the single category of Social. Two-tailed bivariate Pearson correlations were calculated for each psychopathy measure and each semantic feature for the non-clinical data using LIWC. The results are presented in Table 6. However, similar to the analysis with the GI lexicon, no relationships were found to be significant with either $\alpha=0.05$ or $\alpha=0.002$.

\section{Clinical Sample}


Descriptive Analysis. Analysis of the distrbutions of the psychopathy measures and semantic features for the clinical data are presented in Table 7 along with the results from Shapiro-Wilk tests on each variable. For the psychopathy measures, only PCL-YV Factor 2 had a normal distribution while Total PCL-YV and PCL-YV Factor 1 did not have normal distributions. For the semantic features, all had normal distributions except Negative Polarity and Religion words.

Boxplot graphs were then created to determine whether univariate outliers were present in either the psychopathy measures or semantic features (Figure 4). No outliers were found for the clinical psychopathy measures (Figure 4a). However, Figure 4b suggests the presence of seven outliers in the Negative Polarity, Positive Polarity, Arousal, Body, and Social feature data. In order to understand the impact of the outliers, analyses were performed with and without the outliers.

A scatterplot matrix was made of all variables to observe whether relationships or bivariate outliers exist between each psychopathy measure and each semantic feature for the clinical data (Figure 5). The scatterplots of interest are highlighted. Most of the scatterplots suggest a lack of relationship between the variables of interest. However, there appears to be a slight positive linear relationship between Total PCL-YV and Food as well as between PCL-YV Factor 2 and Food. There also seems to be a negative linear trend between PCL-YV Factor 2 and Social words. No bivariate outliers were observed for the clinical scatterplots.

Correlational Analysis. Two-tailed bivariate Pearson correlations were then calculated for each psychopathy measure and each semantic feature for the data with the outliers. The results are presented in Table 8. Using the non-adjusted alpha $\alpha=0.05$, there was a significant positive 
Table 7

Mean (M), standard deviation (SD), skew, kurtosis, and Shapiro-Wilk statistic and its p-value for linguistic features and clinical psychopathy scores

\begin{tabular}{lcccccc}
\hline Attribute & $M$ & $S D$ & Skew & Kurtosis & $\begin{array}{c}\text { Shapiro-Wilk } \\
\text { Statistic }\end{array}$ & P-value \\
\hline PCL-YV & 17.54 & 8.37 & 0.51 & -0.91 & 0.91 & 0.032 \\
PCL-YV F1 & 6.04 & 4.00 & 0.53 & -1.01 & 0.91 & 0.023 \\
PCL-YV F2 & 10.26 & 4.69 & 0.18 & -1.20 & 0.92 & 0.061 \\
Negative & 0.15 & 0.02 & 0.97 & 1.13 & 0.90 & 0.019 \\
Polarity & & & & & & \\
Positive & 0.16 & 0.04 & 1.15 & 1.67 & 0.92 & 0.051 \\
Polarity & & & & & & \\
Arousal & 0.85 & 0.13 & 2.18 & 6.33 & 0.97 & 0.644 \\
Body & 0.01 & 0.004 & 0.48 & 0.10 & 0.97 & 0.685 \\
Food & 0.01 & 0.004 & 0.64 & -0.62 & 0.93 & 0.094 \\
Social & 0.02 & 0.008 & 0.98 & 1.01 & 0.93 & 0.110 \\
Religion & 0.002 & 0.002 & 1.01 & 0.99 & 0.89 & 0.013 \\
\hline
\end{tabular}
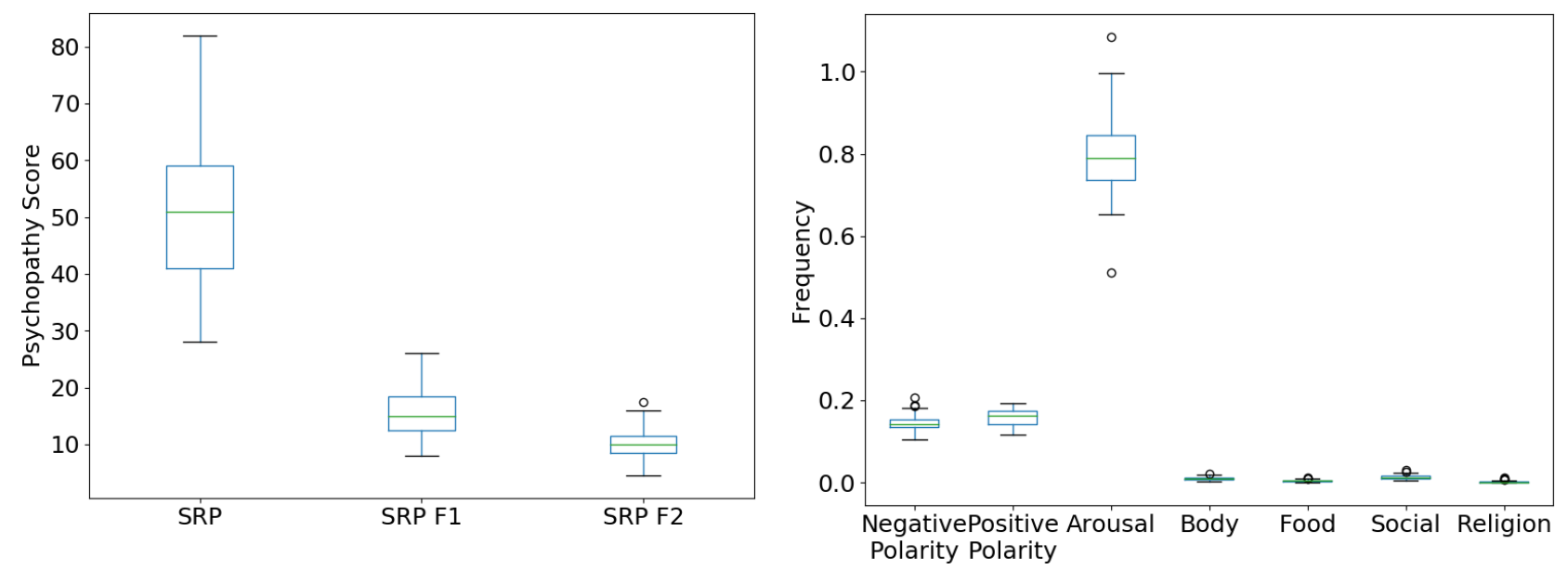

a)

b)

Figure 4. Boxplots for a) psychopathy scales and b) semantic features for clinical data with univariate outliers indicated 


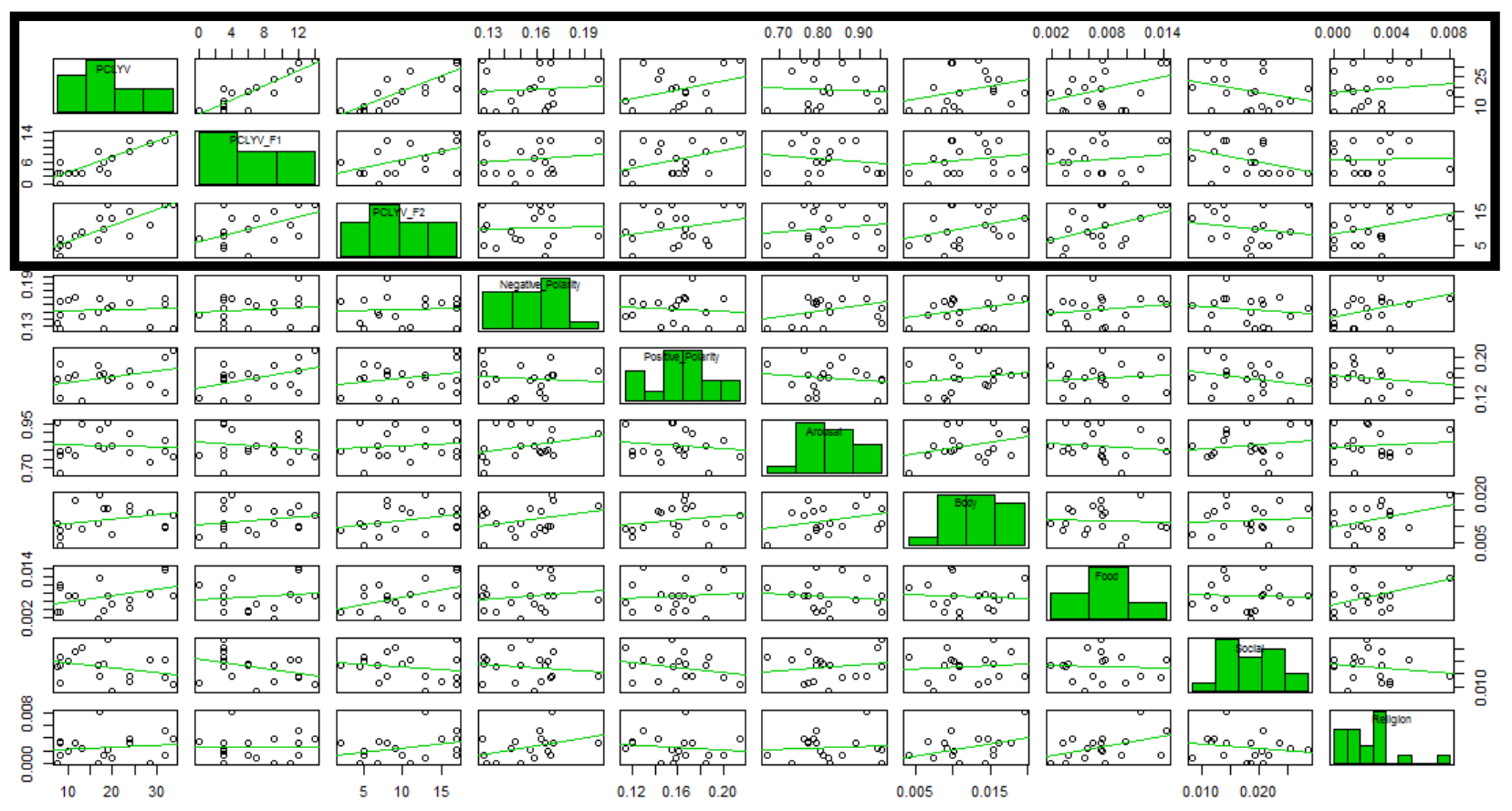

Figure 5. Scatterplot matrix of psychopathy scales and semantic features for clinical data with univariate outliers.

Table 8

Correlation coefficients ( $r$ ) and p-values (p) for two-tailed bivariate Pearson correlation between psychopathy measures and linguistic features for clinical data, $*=$ significant at $\alpha=0.05, * *=$ significant at $\alpha=0.002$

\begin{tabular}{lcccccc}
\hline & \multicolumn{2}{c}{ Total PCL-YV } & \multicolumn{2}{c}{ PCL-YV Factor 1 } & \multicolumn{2}{c}{ PCL-YV Factor 2 } \\
\cline { 2 - 7 } Attributes & $r$ & $p$ & $r$ & $p$ & $r$ & $p$ \\
\hline $\begin{array}{l}\text { Negative } \\
\text { Polarity }\end{array}$ & 0.19 & 0.370 & 0.18 & 0.398 & 0.20 & 0.340 \\
$\begin{array}{l}\text { Positive } \\
\text { Polarity }\end{array}$ & 0.20 & 0.349 & 0.13 & 0.527 & 0.17 & 0.419 \\
Arousal & & & & & & \\
Body & -0.01 & 0.959 & -0.15 & 0.467 & 0.22 & 0.299 \\
Food & 0.35 & 0.085 & 0.28 & 0.180 & 0.30 & 0.149 \\
Social & $0.47^{*}$ & 0.018 & 0.19 & 0.377 & $0.57^{*}$ & 0.003 \\
Religion & $-0.46^{*}$ & 0.022 & $-0.44^{*}$ & 0.027 & -0.33 & 0.107 \\
\hline
\end{tabular}




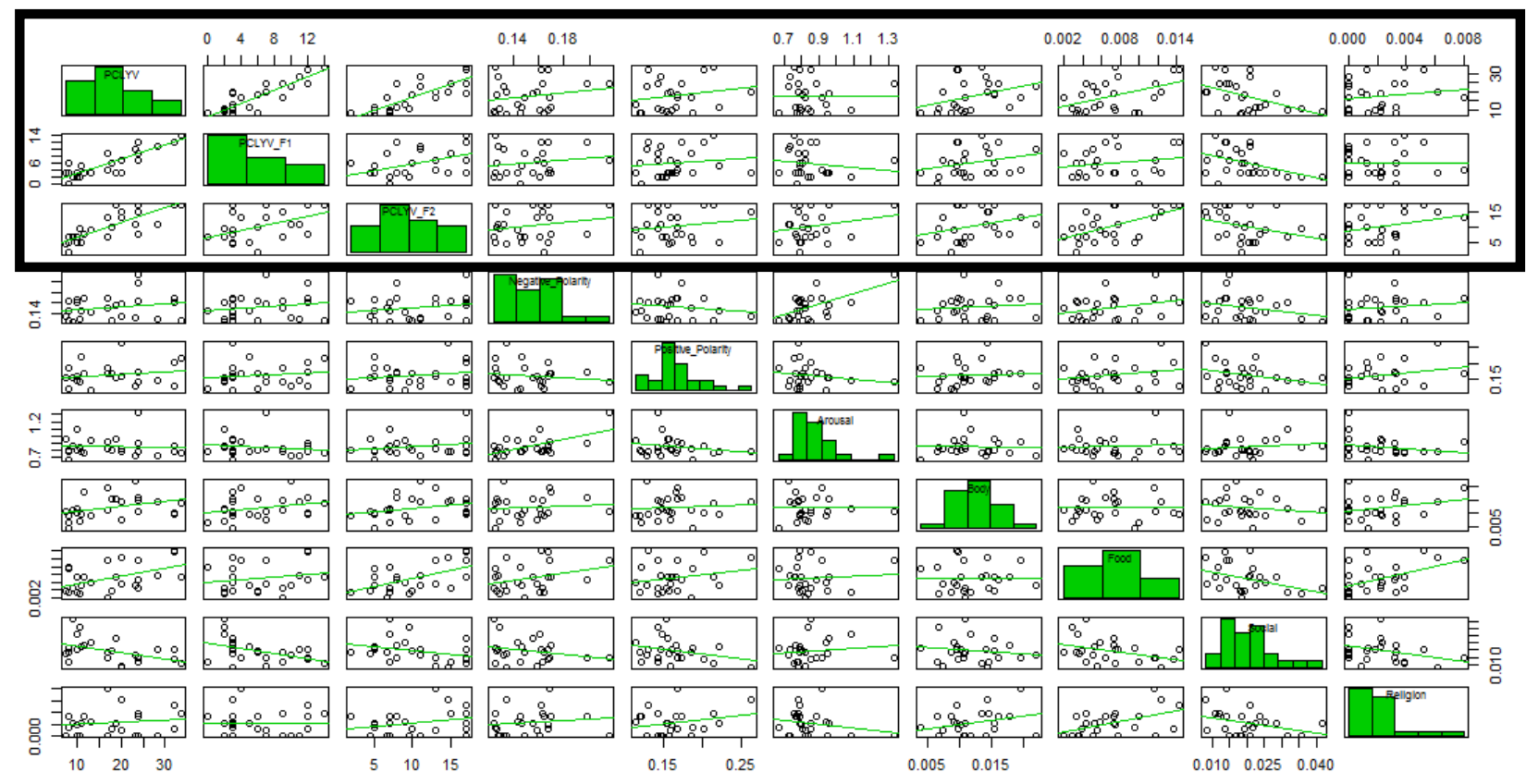

Figure 6. Scatterplot matrix of psychopathy scales and semantic features for clinical data without univariate outliers.

Table 9

Correlation coefficients ( $r$ ) and p-values (p) for two-tailed bivariate Pearson correlation between psychopathy measures and linguistic features for clinical data excluding univariate outliers, $*=$ significant at $\alpha=0.05, * *=$ significant at $\alpha=0.002$

\begin{tabular}{lcccccc}
\hline & \multicolumn{2}{c}{ Total PCL-YV } & \multicolumn{2}{c}{ PCL-YV Factor 1 } & \multicolumn{2}{c}{ PCL-YV Factor 2 } \\
\cline { 2 - 7 } Attributes & $r$ & $p$ & $r$ & $p$ & $r$ & $p$ \\
\hline $\begin{array}{l}\text { Negative } \\
\text { Polarity }\end{array}$ & 0.16 & 0.486 & 0.21 & 0.367 & 0.08 & 0.739 \\
Positive & 0.39 & 0.078 & 0.44 & $0.045^{*}$ & 0.27 & 0.233 \\
Polarity & & & & & & \\
Arousal & -0.07 & 0.787 & -0.18 & 0.480 & 0.13 & 0.599 \\
Body & 0.33 & 0.150 & 0.24 & 0.305 & 0.32 & 0.156 \\
Food & $0.47^{*}$ & 0.032 & 0.27 & 0.231 & 0.51 & 0.019 \\
Social & -0.29 & 0.244 & -0.36 & 0.138 & -0.18 & 0.476 \\
Religion & 0.23 & 0.313 & 0.14 & 0.549 & 0.33 & 0.140 \\
\hline
\end{tabular}


Table 10

Correlation coefficients (r) and p-values (p) for two-tailed bivariate Pearson correlation between psychopathy measures and linguistic features for clinical data with swear words included, * = significant at $\alpha=0.05, * *=$ significant at $\alpha=0.002$

\begin{tabular}{lcccccc}
\hline & \multicolumn{2}{c}{ Total PCL-YV } & \multicolumn{2}{c}{ PCL-YV Factor 1 } & \multicolumn{2}{c}{ PCL-YV Factor 2 } \\
\cline { 2 - 7 } Attributes & $r$ & $p$ & $r$ & $p$ & $r$ & $p$ \\
\hline $\begin{array}{l}\text { Negative } \\
\text { Polarity }\end{array}$ & 0.15 & 0.468 & 0.14 & 0.513 & 0.18 & 0.390 \\
$\begin{array}{l}\text { Positive } \\
\text { Polarity }\end{array}$ & 0.19 & 0.360 & 0.13 & 0.538 & 0.17 & 0.430 \\
Arousal & & & & & & \\
Body & -0.04 & 0.833 & -0.19 & 0.369 & 0.19 & 0.356 \\
Food & 0.35 & 0.090 & 0.28 & 0.183 & 0.29 & 0.155 \\
Social & $0.46^{*}$ & 0.021 & 0.17 & 0.413 & $0.57^{*}$ & 0.003 \\
Religion & -0.13 & 0.526 & -0.21 & 0.323 & -0.02 & 0.916 \\
& 0.14 & 0.495 & -0.01 & 0.970 & 0.26 & 0.210 \\
\hline
\end{tabular}

Table 11

Correlation coefficients $(r)$ and p-values $(p)$ for two-tailed bivariate Pearson correlation between psychopathy measures and linguistic features for clinical data using LIWC, * = significant at $\alpha=0.05, * *=$ significant at $\alpha=0.002$

\begin{tabular}{lcccccc}
\hline & \multicolumn{2}{c}{ Total PCL-YV } & \multicolumn{2}{c}{ PCL-YV Factor 1 } & \multicolumn{2}{c}{ PCL-YV Factor 2 } \\
\cline { 2 - 7 } Attributes & $r$ & $P$ & $r$ & $P$ & $r$ & $p$ \\
\hline $\begin{array}{l}\text { Negative } \\
\text { Polarity }\end{array}$ & $0.43^{*}$ & 0.032 & $0.40^{*}$ & 0.046 & 0.33 & 0.106 \\
$\begin{array}{l}\text { Positive } \\
\text { Polarity }\end{array}$ & -0.19 & 0.373 & -0.24 & 0.248 & -0.17 & 0.409 \\
Body & & & & & & \\
Food & $0.55^{*}$ & 0.004 & $0.53^{*}$ & 0.007 & $0.47 *$ & 0.018 \\
Family & -0.08 & 0.709 & -0.21 & 0.313 & -0.03 & 0.894 \\
Friend & -0.25 & 0.234 & -0.20 & 0.336 & -0.15 & 0.482 \\
Religion & 0.06 & 0.772 & 0.16 & 0.447 & -0.12 & 0.568 \\
\hline
\end{tabular}


relationship between Total PCL-YV and Food, $r(25)=0.47, p=0.018$. There was also a significant negative relationship between Total PCL-YV and Social, $r(25)=-0.46, p=0.022$.

The only other negative relationship with the Social category was found with Factor 1 of PCL$\mathrm{YV}, r(25)=-0.44, p=0.027$. In addition, the only other positive relationship with Food was found with Factor 2 of PCL-YV, $r(25)=0.57, p=0.003$. All relationships were of medium strength. However, using the Bonferroni correction, these relationships were all no longer found to be significant.

A scatterplot matrix was then made again of the clinical data excluding the outliers (Figure 6). Once again, excluding these outliers reduced variability in the data. The resulting matrix suggest that there may be a weak positive relationship between Total PCL-YV and Negative Polarity that was not apparent in the scatter matrix with outliers in. In addition, the positive relationship between Total PCL-YV and Food words, and the negative relationship between PCL-YV Factor 2 and Social words found in Figure 5 are still present in Figure 6.

Two-tailed bivariate Pearson correlations were again performed between each psychopathy measure and each semantic feature for the data without the outliers. The results are presented in Table 9. Using $\alpha=0.05$, only the relationship between Total PCL-YV and Food remained significant, $r(21)=0.47, p=0.032$. In addition, the relationship between Factor 1 and Positive Polarity becomes statistically significant, $r(21)=0.44, p=0.045$. Once again, however, once the alpha was corrected, none of the correlations were found significant.

In order to determine whether swear words had an effect on analysis, a final analysis was performed with swear words included. Two-tailed bivariate Pearson correlations were calculated for each psychopathy measure and each semantic feature for the clinical data with swear words. 
The results are presented in Table 10. None of the relationships were found to be significant using either $\alpha=0.05$ or $\alpha=0.002$.

Finally, semantic categories were once again extracted using LIWC2015 (Pennebaker, Boyd, Jordan, \& Blackburn, 2015). Two-tailed bivariate Pearson correlations were calculated for each psychopathy measure and each semantic feature for the clinical data using LIWC. The results are presented in Table 11. Using $\alpha=0.05$, a significant positive relationship was found between Total PCL-YV and Negative Polarity, $r(25)=0.43, p=0.032$. A significant positive relationship was also found between Total PCL-YV and Body words, $r(25)=0.55, p=0.004$. Significant positive relationships were also found between Factor 1 and Negative Polarity $(r(25)$ $=0.40, p=0.046)$, as well as Factor 1 and Body words $(r(25)=0.53, p=0.007)$. However, for Factor 2, only a relationship with Body words was found to be significant, $r(25)=0.47, p=$ 0.018. As with previous analyses, however, these relationships were no longer significant once the alpha was adjusted using the Bonferroni adjustment.

\section{General Discussion}

I analyzed the relationship between psychopathy measures and emotional polarity for both a non-clinical and clinical sample. In addition, psychopathy measures and semantic categories belonging to Maslow's hierarchy of needs (1943) were analyzed for both samples. The results of these analyses and how they relate to previous findings are discussed below.

\section{Non-Clinical Sample}

Psychopathy and Sentiment. I hypothesized that non-clinical psychopathy scores would be related to sentiment polarity and strength, particularly negative polarity. In addition, I predicted that Factor 1 of non-clinical psychopathy scores would be related to emotional polarity. 
However, no significant relationships were found to support these hypotheses. This remained true regardless of whether outliers were included or not, as well as whether swear words were included or not, and which lexicon was used. In addition, regardless of their significance and the presence of outliers, all relationships were weak.

Contrary to the results of the present study, previous research found that non-clinical psychopathy was associated with emotional polarity in text. Previous studies reported a weak positive correlation between negative emotional polarity and non-clinical psychopathy (Sumner et al., 2012; Preotiuc-Pietro et al., 2016). They also reported a weak negative correlation between positive emotional polarity and non-clinical psychopathy (Garcia \& Sikström, 2014; Sumner et al., 2012). Their findings suggest that individuals with high non-clinical psychopathy scores use more negative emotional words and less positive emotional words than those who scored low. Like the present study, however, findings from previous studies suggest that the impact of nonclinical psychopathy on emotional word use was small.

No previous studies have investigated possible relationships between sentiment in transcribed speech and Factor 1 of non-clinical psychopathy. The present study is currently the only one to examine in this relation. Therefore, it is unknown whether the present finding that there is a lack of evidence to support a relationship between emotional polarity and Factor 1 is accurate, in error, or simply due to methodological/statistical issues.

Psychopathy and Maslow's Hierarchy. I predicted that non-clinical psychopathy would be related to Maslow's Hierarchy of needs. Specifically, I hypothesized that individuals who scored high on non-clinical psychopathy would use more words related to physical needs and fewer words related to social needs. None of the relationships between physical or social needs and non-clinical psychopathy measures were found to be significant, however. These included an 
exploration of relationships between Factor 1 and 2 of non-clinical psychopathy and Maslow's Hierarchy of needs. Relationships remained non-significant when outliers or swear words were removed as well. In addition, relationships were non-significant when the LIWC lexicon was used.

My findings are consistent with some elements of previous research, however. Only two studies have explored the relationship between specific semantic categories and non-clinical psychopathy. Both found a significant positive but weak relationship between non-clinical psychopathy and Body words (Sumner et al., 2012; Preotiuc-Pietro et al., 2016). However, only one study found a significant negative weak relationship between non-clinical psychopathy and Family words (Sumner et al., 2012). The latter study also found a weak relationship between non-clinical psychopathy and Friends words, but it was not significant. These studies suggest that individuals that score high on non-clinical psychopathy use more Body words and less Family words. In addition, they suggest that the impact of non-clinical psychopathy on Body and Family words is small, similar to the present study's findings.

As these earlier studies did not explicitly investigate Maslow's hierarchy of needs, they do not report all categories relating to physical and social needs. These researchers could have failed to report categories because they did not investigate them or because the results were nonsignificant. For example, Sumner et al. (2012) reported both significant and non-significant results. Therefore, the reason they did not include Food and Religion words in their report is likely because they did not analyze these categories. However, Preotiuc-Pietro et al. (2016) only reported significant findings. Therefore, it is possible that they investigated possible correlations between non-clinical psychopathy and Food, Social, and Religion word, but they were not significant. If this is the case, Preotiuc-Pietro et al. (2016)'s lack of evidence of these 
relationships corroborates with the present study's findings. Because both Preotiuc-Pietro et al. (2016) and the current study did not find sufficient evidence, it may be possible that non-clinical psychopathy is not related to Food, Social, or Religion words. However, more research on nonclinical psychopathy and the production of Maslow's hierarchy must be performed to determine whether this conclusion is valid.

\section{Clinical Sample}

Psychopathy and Sentiment. As with the non-clinical sample, I predicted that clinical psychopathy would be related to sentiment polarity and strength in text. Factor 1 of clinical psychopathy in particular was hypothesized to be related to emotional polarity. I performed correlational analyses on clinical psychopathy measures and emotional polarity word use. The relationships found were all weak, but non-significant, regardless of whether outliers were removed or not, as well as whether swear words were included or not, or which lexicon was used. Therefore, the results do not provide evidence to support my hypotheses.

To my knowledge, Hancock et al. (2013) is the only study that investigated the relationship between clinical psychopathy and emotional polarity in text. They compared emotional word use between psychopaths and non-psychopaths and found no significant difference between the groups. This parallels the current study's findings of no significant relationships between total clinical psychopathy score and emotional word use. However, Hancock et al. also performed a correlational analysis between clinical psychopathy Factor 1 and positive polarity and found a significant weak negative relationship between the variables. This differs from the current study where I did not find the weak relationship between clinical psychopathy Factor 1 and positive or negative polarity to be significant. 
Psychopathy and Maslow's Hierarchy. The current study hypothesized that clinical psychopathy would also be related Maslow's hierarchy of needs. Correlational analyses were performed on the clinical psychopathy measures and words relating to the hierarchy of needs. Once again, both with and without outliers, with and without swear words, and the GI or LIWC lexicon, all relationships were weak and non-significant. Consistent with the non-clinical sample, no evidence was found to support the existence of these relationships.

Hancock et al. (2013) specifically explored differences in word use related to Maslow's hierarchy of needs between clinical psychopaths and non-psychopaths. For physical needs, they found that psychopaths used significantly more words about Food than non-psychopaths. It appears that they did not measure Body words, although they did find significant differences for other words relating to physical needs such as clothing and money. For social needs, they found that psychopaths used significantly fewer words about Family and Religion than nonpsychopaths. However, unlike the current study, Hancock et al. found enough evidence to suggest an association between clinical psychopathy and most of Maslow's hierarchy of needs.

\section{Limitations}

There are many differences between the current study and previous studies on psychopathy and language that could account for the differences in findings. A likely cause is that the sample size of our study was significantly smaller than in previous studies. All previous studies on nonclinical psychopathy had sample sizes larger than 300 participants. Sumner et al. (2012) recruited almost 3,000 participants through Twitter. These large samples allow for more statistical power in inferential analyses of relationships in the data, especially for non-clinical samples where relationships may not be as strong. Similarly, for clinical psychopathy, the current study had almost half the sample size that Hancock et al. (2013) had. 
Another important difference between the current study and Hancock et al. (2013) is that data for the current study came from institutionalized adolescents while Hancock et al. obtained data from an incarcerated adult prison. Differences in participants includes the severity of crime as the adolescents had only committed assault or robbery, and the adults in Hancock et al. had committed murder. The low severity of crime by adolescents may also be associated with the low number of clinical psychopaths found in the current study (16\%). In comparison, 14 of 52 participants in Hancock et al. could be classified as clinical psychopaths (27\%). Therefore, using participants that were adult prisoners and having a higher number of psychopaths may have made it easier for Hancock et al. to find statistical differences.

Data collection and the text itself may have caused differences in findings. The current study used transcribed speech from interviews while most previous studies used text from social media. In addition, text for the current study's clinical sample was transcribed extemporaneously. In studies that used social media, participants were able to write freely on any topic without supervision. Questions used in the interviews for both the non-clinical and clinical samples in the present study may have been too restrictive in topic and did not allow participants to provide sufficient speech for inferential analyses. Furthermore, since interviews were face-toface in the current study, the presence of the researcher may have had elicited socially desirable responses from the participant (Johnson \& Fendrich, 2005). It has been suggested that participants are more likely to provide responses which portray themselves in positive manner when questions are socially sensitive, such as the questions in the current study (King and Brunner, 2000). In addition, it may be that individuals scoring high on psychopathy (clinical or non-clinical) are more likely to provide socially desirable responses than those low on psychopathy. This is possible as psychopaths are known for manipulating and persuading others 
(Hancock et al., 2013; Hare \& Neumann, 2008). Therefore, these differences in source and production context could also have led to qualitative differences in text between the current study and previous studies.

Differences in linguistic tool may have also affected the results of the present study. The current study used GI lexicon for both sentiment and semantic analysis, and ANEW only for sentiment arousal analysis. GI has been used for sentiment analysis (Hu, Tang, Gao, \& Liu, 2013; Kennedy \& Inkpen, 2005). However, the GI lexicon is built from formal texts and therefore may not perform well on the informal language found in the current study (Stone et al., 1966). In addition, none of the previous studies on psychopathy and language used GI. Sumner et al. (2012) and Preotiuc-Petro et al. (2016) both used LIWC, which is commonly used to investigate sentiment in psychology. Garcia and Sikstrom (2014) used ANEW for both sentiment polarity and arousal, while the current study only used it for arousal. In addition, Hancock et al. (2013) used Wmatrix and found some relationships between sentiment and psychopathy, suggesting it may also be more appropriate than GI for determining sentiment for psychopathy research. The current study, however, found that using the LIWC lexicon did not greatly alter correlational results. In addition, incarceration in the clinical sample may have provided a confound for using Maslow's hierarchy of needs as a linguistic measure. This is because incarcerated adults have been reported to focus on low level physical needs more than social needs (Kasser, 1996). However, it is unknown if this focus on low level needs is also present in incarcerated adolescents.

Finally, the method of determining statistical significance may have been a limitation. The current study used Bonferroni corrections to control Type I error when evaluating the statistical significance of many correlations at one time. However, some researchers have 
suggested that Bonferroni is overly conservation and increases the likelihood of Type II error. Therefore, performing such a conservative correction might have caused a Type II error so that relationships that are true are falsely not be significant. In addition, to my knowledge, it is unknown whether previous studies used Bonferroni or similar correction procedures or not. If they did not use a procedure to control for making multiple comparisons, the significant effects they found may have been artefactual.

\section{Conclusion}

Overall, I did not find strong evidence linking psychopathy to language in the present study. In particular, I failed to replicate a relationship between emotion production in speech content and psychopathy that had been found in four previous studies. Although this relationship between negative sentiment and psychopathy had been consistently observed in individuals with non-clinical psychopathy, Hancock et al. (2013) did not find this relation for total psychopathy score. Therefore, it is a possibility that the emotion deficits of psychopathy are not consistently reflected in speech or text content, especially for those with clinical psychopathy. This suggests that even if deficits in textual emotion production exist for psychopathy, they are not strongly prevalent. Sufficient evidence was also unavailable from the current study to provide confirmation of whether psychopathy is related to Maslow's hierarchy of needs. Again, only four previous studies have been done on this topic and findings have been variable. Therefore, it may be possible that semantic markers do not exist, or if they do, are weak.

Despite the uncertain findings, the current study contributes to literature on personality and language. It is one the few studies focusing on the intersection of language and psychopathy as well as emotion production and psychopathy. Moreover, it is one of only two studies in these areas that include data from individuals with clinical psychopathy. It is also the only study on 
emotion and psychopathy that explored emotion intensity. In addition, this study provides insight into sources of data for text mining in psychology. The restrictive nature of interviews may not allow for sufficient or appropriate semantic information when using text mining in behavioural studies compared to using text derived directly from large numbers of social media users, for example.

Given the difference in findings between the current study and previous research, further work should be done to confirm/disconfirm possible relationships between emotion production and psychopathy and between semantic content and psychopathy. Future work should use large samples in order to have sufficient inferential power, particularly for non-clinical populations, as sample size appears to play a large role in determining whether statistically significant relationships are observed. Based on the current study, it appears that to find significant results in linguistic analyses, the non-clinical sample size should be more than 50. Previous studies suggest that the sample size should at least be 300 . However, future studies should explore a range sample sizes to determine if a size lower than 300 can be used. In addition, it is possible that social media posts allow for more accurate representations of the full range of participants' language use compared to other sources of texts. Future work should explore various sources of texts, comparing text from social media posts versus other sources. As only Hancock et al. (2013) and the current study have looked at language and clinical psychopathy, more work in this area is required. Future studies performing multiple correlations should also use lessconservative methods to control Type I error, such as the Sidak method or Dunnett's test. Finally, it would be beneficial for future studies to compare language production from clinical and non-clinical psychopathy in order to understand the differences between the two groups 
better. Such research could potentially contribute to using language to predict psychopathy as well as differentiate clinical from non-clinical psychopathy. 


\section{References}

Agrawal, A., \& An, A. (2012). Unsupervised emotion detection from text using semantic and syntactic relations. In Proceedings of the The 2012 IEEE/WIC/ACM International Joint Conferences on Web Intelligence and Intelligent Agent Technology-Volume 01 (pp. 346353). IEEE Computer Society.

Agarwal, A., Biadsy, F., \& Mckeown, K. R. (2009, March). Contextual phrase-level polarity analysis using lexical affect scoring and syntactic n-grams. In Proceedings of the 12th Conference of the European Chapter of the Association for Computational Linguistics (pp. 24-32). Association for Computational Linguistics.

Alpaydin, E. (2009). Introduction to machine learning, $2^{\text {nd }}$ ed. Cambridge, MA: MIT Press.

Aman, S., \& Szpakowicz, S. (2007). Identifying expressions of emotion in text. Text, speech and dialogue, 196-205.

Aue, A., \& Gamon, M. (2005). Customizing sentiment classifiers to new domains: A case study. In Proceedings of recent advances in natural language processing (RANLP).

Babiak, P., \& Hare, R. (2007). Snakes in suits: When psychopaths go to work. Toronto, Canada: Harper Collins.

Balahur, A., Hermida, J. M., \& Montoyo, A. (2012). Building and exploiting emotinet, a knowledge base for emotion detection based on the appraisal theory model. IEEE Transactions on Affective Computing, 3(1), 88-101.

Barrett, L. F. (2006). Are Emotions Natural Kinds? Perspectives on psychological science, 1(1), 28-58. 
Baston, C. D., Shaw, L. L., \& Oleson, K. C. (1992). Differentiation affect, mood and emotion. Review of Personality and Social Psychology, 13, 294-326.

Benning, S. D., Patrick, C. J., Hicks, B. M., Blonigen, D. M., \& Krueger, R. F. (2003). Factor structure of the psychopathic personality inventory: validity and implications for clinical assessment. Psychological assessment, 15(3), 340.

Bird, Steven, Edward Loper and Ewan Klein (2009), Natural Language Processing with Python. O’Reilly Media Inc.

Blair, J., Mitchell, D., \& Blair, K. (2005). The psychopath. Blackwell Pub.

Blair, K. S., Richell, R. A., Mitchell, D. G. V., Leonard, A., Morton, J., \& Blair, R. J. R. (2006). They know the words, but not the music: affective and semantic priming in individuals with psychopathy. Biological psychology, 73(2), 114-123.

Bollen, J., Mao, H., \& Pepe, A. (2011). Modeling public mood and emotion: Twitter sentiment and socio-economic phenomena. ICWSM, 11, 450-453.

Bolt, D. M., Hare, R. D., \& Neumann, C. S. (2007). Score Metric Equivalence of the Psychopathy Checklist-Revised (PCL-R) Across Criminal Offenders in North America and the United Kingdom A Critique of Cooke, Michie, Hart, and Clark (2005) and New Analyses. Assessment, 14(1), 44-56.

Bradley, M. M., \& Lang, P. J. (1999). Affective norms for English words (ANEW): Instruction manual and affective ratings (pp. 1-45). Technical report C-1, the center for research in psychophysiology, University of Florida. 
Brandt, J. R., Kennedy, W. A., Patrick, C. J., \& Curtin, J. J. (1997). Assessment of psychopathy in a population of incarcerated adolescent offenders. Psychological Assessment, 9, 429 435.

Cambria, E., Olsher, D., \& Rajagopal, D. (2014). SenticNet 3: a common and common-sense knowledge base for cognition-driven sentiment analysis. In Twenty-eighth AAAI conference on artificial intelligence.

Campbell, M. A., Porter, S., \& Santor, D. (2004). Psychopathic traits in adolescent offenders: An evaluation of criminal history, clinical, and psychosocial correlates. Behavioral sciences \& the law, 22(1), 23-47.

Catchpole, R. E., \& Gretton, H. M. (2003). The predictive validity of risk assessment with violent young offenders: A 1-year examination of criminal outcome. Criminal Justice and Behavior, 30(6), 688-708.

Cambria, E., \& Hussain, A. (2012). Sentic Computing: Techniques, tools, and applications (Vol. 2). Springer Science \& Business Media.

Cambria, E., Schuller, B., Xia, Y., \& Havasi, C. (2013). New avenues in opinion mining and sentiment analysis. IEEE Intelligent Systems, 28(2), 15-21.

Cattell, R. (2006). An introduction to mind, consciousness and language. A\&C Black.

Chaovalit, P., \& Zhou, L. (2005). Movie review mining: A comparison between supervised and unsupervised classification approaches. In System Sciences, 2005. HICSS'05. Proceedings of the 38th Annual Hawaii International Conference on (pp. 112c-112c). IEEE. 
Cleckley, H. (1941). The mask of sanity; an attempt to reinterpret the so-called psychopathic personality.

Cleckley, H. (1976). The mask of sanity. St. Louis: Mosby.

Cleckley, H. M. (1988). The mask of sanity: An attempt to clarify some issues about the so called psychopathic personality, Emily S. Cleckley, Publisher.

Colman, A. M. (2015). A dictionary of psychology. Oxford University Press, USA.

Cooke, D. J., Michie, C., Hart, S. D., \& Clark, D. (2005). Assessing psychopathy in the UK: concerns about cross-cultural generalisability. The British Journal of Psychiatry, 186(4), $335-341$.

Costa Jr, P. T., \& McCrae, R. R. (1990). Personality disorders and the five-factor model of personality. Journal of personality disorders, 4(4), 362-371.

Costa, P. T., \& MacCrae, R. R. (1992). Revised NEO personality inventory (NEO PI-R) and NEO five-factor inventory (NEO-FFI): Professional manual. Psychological Assessment Resources, Incorporated.

Crossley, S. A., Kyle, K., \& McNamara, D. S. (2017). Sentiment Analysis and Social Cognition Engine (SEANCE): An automatic tool for sentiment, social cognition, and social-order analysis. Behavior research methods, 49(3), 803-821.

Dawel, A., O’Kearney, R., McKone, E., \& Palermo, R. (2012). Not just fear and sadness: Metaanalytic evidence of pervasive emotion recognition deficits for facial and vocal expressions in psychopathy. Neuroscience \& Biobehavioral Reviews, 36(10), 2288-2304. 
Deerwester, S., Dumais, S. T., Furnas, G. W., Landauer, T. K., \& Harshman, R. (1990). Indexing by latent semantic analysis. Journal of the American society for information science, 41(6), 391.

DeMatteo, D., Heilbrun, K., \& Marczyk, G. (2006). An empirical investigation of psychopathy in a noninstitutionalized and noncriminal sample. Behavioral sciences \& the law, 24(2), 133-146.

Derefinko, K., \& Lynam, D. R. (2013). Psychopathy from the perspective of the five-factor model of personality.

Dey, L., \& Haque, S. M. (2009). Opinion mining from noisy text data. International Journal on Document Analysis and Recognition (IJDAR), 12(3), 205-226.

Digman, J. M. (1990). Personality structure: Emergence of the five-factor model. Annual review of psychology, 41(1), 417-440.

Ding, X., Liu, B., \& Yu, P. S. (2008). A holistic lexicon-based approach to opinion mining. In Proceedings of the 2008 international conference on web search and data mining (pp. 231-240). ACM.

Dotterer, H. L., Waller, R., Neumann, C. S., Shaw, D. S., Forbes, E. E., Hariri, A. R., \& Hyde, L. W. (2016). Examining the Factor Structure of the Self-Report of Psychopathy Short-Form Across Four Young Adult Samples. Assessment. 
Edens, J. F., Marcus, D. K., Lilienfeld, S. O., \& Poythress Jr, N. G. (2006). Psychopathic, not psychopath: taxometric evidence for the dimensional structure of psychopathy. Journal of abnormal psychology, 115(1), 131.

Ekman, Paul. "Facial expressions." Handbook of cognition and emotion 16 (1999): 301-320.

Esuli, A., \& Sebastiani, F. (2007). SENTIWORDNET: A high-coverage lexical resource for opinion mining. Evaluation, 1-26.

Eysenck, H. J., \& Eysenck, S. B. G. (1991). Manual of Eysenck personality scales (EPS adult): comprising the EPQ-Revised (EPQ-R), EPQ-R short scale, Impulsiveness (IVE) questionnaire. Hodder \& Stoughton.

Farrington, D. P. (2006). Family background and psychopathy. Handbook of psychopathy, 229250.

Feldman Barrett, L., \& Russell, J. A. (1998). Independence and bipolarity in the structure of current affect. Journal of personality and social psychology, 74(4), 967.

Feldman, R., \& Sanger, J. (2007). The text mining handbook: advanced approaches in analyzing unstructured data. Cambridge university press.

Feng, V. W., \& Hirst, G. (2014). A Linear-Time Bottom-Up Discourse Parser with Constraints and Post-Editing. In $A C L$ (1) (pp. 511-521).

Fisher, C. D. (2000). Mood and emotions while working: missing pieces of job satisfaction?. Journal of organizational behavior, 185-202.

Forth, A. E., \& Flight, J. I. (2007). Instrumentally violent youth: The roles of psychopathic traits, empathy, and attachment. Criminal Justice and Behavior. 
Forth, A. E., \& Kosson, D. (2003). The hare PCL: youth version. Toronto, ON: Multi-Health Systems.

Garcia, D., \& Sikström, S. (2014). The dark side of Facebook: Semantic representations of status updates predict the Dark Triad of personality. Personality and Individual Differences, 67, 92-96.

Gohm, C. L., \& Clore, G. L. (2002). Four latent traits of emotional experience and their involvement in well-being, coping, and attributional style. Cognition \& Emotion, 16(4), 495-518.

Gooty, J., Connelly, S., Griffith, J., \& Gupta, A. (2010). Leadership, affect and emotions: A state of the science review. The Leadership Quarterly, 21(6), 979-1004.

Gordon, S. L. (1981). The sociology of sentiments and emotion. Social psychology: Sociological perspectives, 562-592.

Gordts, S., Uzieblo, K., Neumann, C., Van den Bussche, E., \& Rossi, G. (2015). Validity of the Self-Report Psychopathy Scales (SRP-III full and short versions) in a community sample. Assessment.

Gosling, S. D., Rentfrow, P. J., \& Swann, W. B. (2003). A very brief measure of the Big-Five personality domains. Journal of Research in personality, 37(6), 504-528.

Guay, J. P., Ruscio, J., Knight, R. A., \& Hare, R. D. (2007). A taxometric analysis of the latent structure of psychopathy: evidence for dimensionality. Journal of Abnormal Psychology, 116(4), 701. 
Gustafson, S. B., \& Ritzer, D. R. (1995). The dark side of normal: A psychopathy-linked pattern called aberrant self-promotion. European Journal of Personality, 9(3), 147-183.

Hancock, J. T., Woodworth, M. T., \& Porter, S. (2013). Hungry like the wolf: A word-pattern analysis of the language of psychopaths. Legal and Criminological Psychology, 18(1), 102-114.

Hare, R. D. (1980). A research scale for the assessment of psychopathy in clinical population. Personality and Individual Differences, 1(2), 111-119.

Hare, R. D. (1982). Psychopathy and the personality dimensions of psychoticism, extraversion and neuroticism. Personality and Individual Differences, 3(1), 35-42.

Hare, R. D., Hart, S. D., \& Harpur, T. J. (1991). Psychopathy and the DSM-IV criteria for antisocial personality disorder. Journal of abnormal psychology, 100(3), 391.

Hare, R. D. (2003). The psychopathy checklist-Revised. Toronto: ON.

Hare, R.D., \& Neumann, C.S. (2006). The PCL-R assessment of psychopathy: Development, structural properties, and new directions. In C. Patrick (Ed.), Handbook of Psychopathy (pp. 58-90). New York: The Guilford Press.

Hare, R. D., \& Neumann, C. S. (2008). Psychopathy as a clinical and empirical construct. The Annual Review of Clinical Psychology, 4, 217-246.

Harpur, T. J., Hart, S. D., \& Hare, R. D. (2002). Personality of the psychopath. In P. T. Costa \& T. A. Widiger (Eds.), Personality disorders and the five-factor model of personality, $2^{\text {nd }}$ ed. (pp. 299-324). Washigton, DC: American Psychological Association. 
Hart, S. D., Cox, D. N., \& R D. Hare. (1995). The Hare psychopathy checklist: Screening version (PCL: SV). MSH-Multi-Health Systems, Incorporated.

Hart, S. D., \& Hare, R. D. (1994). Psychopathy and the Big 5: Correlations between observers' ratings of normal and pathological personality. Journal of Personality Disorders, 8(1), 3240.

Hatzivassiloglou, V., \& Wiebe, J. M. (2000). Effects of adjective orientation and gradability on sentence subjectivity. In Proceedings of the 18th conference on Computational linguistics, Vol. 1 (pp. 299-305). Association for Computational Linguistics.

Hirsh, J. B., \& Peterson, J. B. (2009). Personality and language use in self-narratives. Journal of research in personality, 43(3), 524-527.

Hu, M., \& Liu, B. (2004). Mining and summarizing customer reviews. In Proceedings of the tenth ACM SIGKDD international conference on Knowledge discovery and data mining (pp. 168-177). ACM.

Hutto, C. J., \& Gilbert, E. (2014). Vader: A parsimonious rule-based model for sentiment analysis of social media text. In Eighth International AAAI Conference on Weblogs and Social Media (ICWSM).

Hutto, C. J., Yardi, S., \& Gilbert, E. (2013). A longitudinal study of follow predictors on twitter. In Proceedings of the sigchi conference on human factors in computing systems (pp. 821830). ACM.

Ignatow, G., \& Mihalcea, R. (2016). Text Mining: A Guidebook for the Social Sciences. Sage Publications. 
Izard, C. E. (2010). The many meanings/aspects of emotion: Definitions, functions, activation, and regulation. Emotion Review, 2(4), 363-370.

Jonason, P. K., \& Webster, G. D. (2010). The dirty dozen: A concise measure of the dark triad. Psychological assessment, 22(2), 420.

Jones, D. N., \& Paulhus, D. L. (2014). Introducing the short dark triad (SD3) a brief measure of dark personality traits. Assessment, 21(1), 28-41.

Kelly, J. R., \& Barsade, S. G. (2001). Mood and emotions in small groups and work teams. Organizational behavior and human decision processes, 86(1), 99-130.

Kennedy, A., \& Inkpen, D. (2006). Sentiment classification of movie reviews using contextual valence shifters. Computational intelligence, 22(2), 110-125.

Kleinginna, P. R., \& Kleinginna, A. M. (1981). A categorized list of emotion definitions, with suggestions for a consensual definition. Motivation and emotion, 5(4), 345-379.

Landauer, T. K., \& Dumais, S. T. (1997). A solution to Plato's problem: The latent semantic analysis theory of acquisition, induction, and representation of knowledge. Psychological review, 104(2), 211.

Lasswell, H. D., \& Namenwirth, J. Z. (1969). The Lasswell value dictionary. New Haven.

LeBreton, J. M., Binning, J. F., \& Adorno, A. J. (2006). Subclinical psychopaths. Comprehensive handbook of personality and psychopathology, 1, 388-411. 
Lee, K., Ashton, M. C., Wiltshire, J., Bourdage, J. S., Visser, B. A., \& Gallucci, A. (2013). Sex, power, and money: Prediction from the Dark Triad and Honesty-Humility. European Journal of Personality, 27(2), 169-184.

Leistico, A. M., Salekin, R. T., DeCoster, J., \& Rogers, R. (2008). A large-scale meta-analysis relating the hare measures of psychopathy to antisocial conduct. Law and Human Behavior, 32, $28-45$.

Levenson, M. R. (1992). Rethinking psychopathy. Theory \& Psychology, 2(1), 51-71.

Lilienfeld, S. O. (1994). Conceptual problems in the assessment of psychopathy. Clinical Psychology Review, 14(1), 17-38.

Liu, B. (2012). Sentiment analysis and opinion mining. Synthesis lectures on human language technologies, 5(1), 1-167.

Liu, B. (2015). Sentiment analysis: Mining opinions, sentiments, and emotions. Cambridge University Press.

Long, L. S., \& Titone, D. A. (2007). Psychopathy and verbal emotion processing in nonincarcerated males. Cognition and Emotion, 21(1), 119-145.

Louth, S. M., Williamson, S., Alpert, M., Pouget, E. R., \& Hare, R. D. (1998). Acoustic distinctions in the speech of male psychopaths. Journal of Psycholinguistic Research, 27(3), 375-384.

Lykken, D. T. (1995). The antisocial personalities. Psychology Press.

Lynam, D. R. (2002). Fledgling psychopathy: A view from personality theory. Law and Human Behavior, 26(2), 255-259. 
Mahmut, M. K., Menictas, C., Stevenson, R. J., \& Homewood, J. (2011). Validating the factor structure of the Self-Report Psychopathy scale in a community sample. Psychological Assessment, 23, 670-678.

Maples, J. L., Lamkin, J., \& Miller, J. D. (2014). A test of two brief measures of the dark triad: The dirty dozen and short dark triad. Psychological assessment, 26(1), 326.

Martin, E. (2015). Concise medical dictionary. Oxford University Press, USA.

Maslow, A. H. (1943). A theory of human motivation. Psychological Review, 50, 370-396.

Mehl, M. R., \& Gill, A. J. (2010). Computerized content analysis. Advanced methods for behavioral research on the internet. Washington DC: American Psychological Association Publications.

Mihalcea, R., \& Pulman, S. (2007). Characterizing humour: An exploration of features in humorous texts. In International Conference on Intelligent Text Processing and Computational Linguistics (pp. 337-347). Springer Berlin Heidelberg.

Miller, G. A., Beckwith, R., Fellbaum, C., Gross, D., \& Miller, K. J. (1990). Introduction to WordNet: An on-line lexical database. International journal of lexicography, 3(4), 235 244.

Mohammad, S. M., \& Turney, P. D. (2010). Emotions evoked by common words and phrases: Using Mechanical Turk to create an emotion lexicon. In Proceedings of the NAACL HLT 2010 workshop on computational approaches to analysis and generation of emotion in text, 26-34. 
Mohammad, S. M., \& Turney, P. D. (2013). Crowdsourcing a word-emotion association lexicon. Computational Intelligence, 29(3), 436-465.

Mudinas, A., Zhang, D., \& Levene, M. (2012, August). Combining lexicon and learning based approaches for concept-level sentiment analysis. In Proceedings of the First International Workshop on Issues of Sentiment Discovery and Opinion Mining (p. 5). ACM.

Munezero, M. D., Montero, C. S., Sutinen, E., \& Pajunen, J. (2014). Are they different? affect, feeling, emotion, sentiment, and opinion detection in text. IEEE transactions on affective computing, 5(2), 101-111.

Neal, T. M. S., \& Sellbom, M. (2012). Examining the factor structure of the Hare Self-Report Psychopathy Scale. Journal of Personality Assessment, 94, 244-253.

Neumann, C. S., Kosson, D. S., Forth, A. E., \& Hare, R. D. (2006). Factor structure of the Hare Psychopathy Checklist: Youth Version (PCL: YV) in incarcerated adolescents. Psychological assessment, 18(2), 142.

Neumann, C. S., Hare, R. D., \& Newman, J. P. (2007). The super-ordinate nature of the Psychopathy Checklist-Revised. Journal of personality disorders, 21(2), 102-117.

O'Connor, B., Balasubramanyan, R., Routledge, B. R., \& Smith, N. A. (2010). From tweets to polls: Linking text sentiment to public opinion time series. ICWSM, 11, 122-129.

Oberlander, J., \& Nowson, S. (2006, July). Whose thumb is it anyway?: classifying author personality from weblog text. In Proceedings of the COLING/ACL on Main conference poster sessions (pp. 627-634). Association for Computational Linguistics. 
O'Connor, B. P. (2005). A search for consensus on the dimensional structure of personality disorders. Journal of Clinical Psychology, 61(3), 323-345.

Pang, B., Lee, L., \& Vaithyanathan, S. (2002). Thumbs up?: Sentiment classification using machine learning techniques. In Proceedings of the ACL-02 conference on Empirical methods in natural language processing-Volume 10 (pp. 79-86). Association for Computational Linguistics.

Pang, B., \& Lee, L. (2005). Seeing stars: Exploiting class relationships for sentiment categorization with respect to rating scales. In Proceedings of the 43rd annual meeting on association for computational linguistics (pp. 115-124). Association for Computational Linguistics.

Pang, B., \& Lee, L. (2008). Opinion mining and sentiment analysis. Foundations and Trends ${ }^{\circledR}$ in Information Retrieval, 2(1-2), 1-135.

Paulhus, D. L., \& Williams, K. M. (2002). The dark triad of personality: Narcissism, Machiavellianism, and psychopathy. Journal of research in personality, 36(6), 556-563.

Paulhus, D. L., Hemphill, J. F., \& Hare, R. D. (2012). Self-report psychopathy scale (SRPIII). Toronto: Multi-Health Systems.

Paulhus, D. L., Neumann, C. S., \& Hare, R. D. (in press). Manual for the Self-Report Psychopathy (SRP) scale. Toronto, Ontario, Canada: Multi-Health Systems.

Pennebaker, J. W., Chung, C. K., Ireland, M., Gonzales, A., \& Booth, R. J. (2007). The development and psychometric properties of LIWC2007: LIWC. net. 
Pennebaker, J. W., Boyd, R. L., Jordan, K., \& Blackburn, K. (2015). The development and psychometric properties of LIWC2015.

Pethman, T. M., \& Erlandsson, S. I. (2002). Aberrant self-promotion or subclinical psychopathy in a Swedish general population. The Psychological Record, 52(1), 33.

Plutchik, R. (1980). Emotion: A psychoevolutionary synthesis. Harpercollins College Division.

Preotiuc-Pietro, D., Carpenter, J., Giorgi, S., \& Ungar, L. (2016). Studying the Dark Triad of Personality through Twitter Behavior. In Proceedings of the 25th ACM International on Conference on Information and Knowledge Management (pp. 761-770). ACM.

Rayson, P. (2008). Wmatrix corpus analysis and comparison tool. Lancaster University.

Ribeiro, F. N., Araújo, M., Gonçalves, P., Gonçalves, M. A., \& Benevenuto, F. (2016). SentiBench-a benchmark comparison of state-of-the-practice sentiment analysis methods. EPJ Data Science, 5(1), 1-29.

Samuel, D. B., \& Widiger, T. A. (2008). A meta-analytic review of the relationships between the five-factor model and DSM-IV-TR personality disorders: A facet level analysis. Clinical psychology review, 28(8), 1326-1342.

Scherer, K. R. (2000). Psychological models of emotion. The neuropsychology of emotion, 137(3), 137-162.

Scherer, K. R. (2005). What are emotions? And how can they be measured?. Social science information, 44(4), 695-729.

Schouten, K., \& Frasincar, F. (2016). Survey on aspect-level sentiment analysis. IEEE Transactions on Knowledge and Data Engineering, 28(3), 813-830. 
Schmidhuber, J. (2015). Deep learning in neural networks: An overview. Neural networks, 61, 85-117.

Smith, G. E., \& Summers, M. D. (1978). Analysis of baculovirus genomes with restriction endonucleases. Virology, 89(2), 517-527.

Socher, R., Perelygin, A., Wu, J. Y., Chuang, J., Manning, C. D., Ng, A. Y., \& Potts, C. (2013). Recursive deep models for semantic compositionality over a sentiment treebank. In Proceedings of the conference on empirical methods in natural language processing $(E M N L P)$.

Stavrianou, A., Andritsos, P., \& Nicoloyannis, N. (2007). Overview and semantic issues of text mining. SIGMOD Record, 36(3), 23-34.

Strapparava, C., \& Valitutti, A. (2004). WordNet Affect: An affective extension of WordNet. Proceedings of the 4th International Conference on Language Resources and Evaluation, 4, 1083-1086.

Strapparava, C., \& Mihalcea, R. (2008). Learning to identify emotions in text. Proceedings of the 2008 ACM symposium on Applied computing, 1556-1560

Stone, P. J., Dunphy, D. C., \& Smith, M. S. (1966). The general inquirer: A computer approach to content analysis.

Sumner, C., Byers, A., Boochever, R., \& Park, G. J. (2012, December). Predicting dark triad personality traits from twitter usage and a linguistic analysis of tweets. In Machine learning and applications (ICMLA), 2012 11th international conference on. IEEE. 
Taboada, M., Brooke, J., Tofiloski, M., Voll, K., \& Stede, M. (2011). Lexicon-based methods for sentiment analysis. Computational linguistics, 37(2), 267-307.

Tausczik, Y. R., \& Pennebaker, J. W. (2010). The psychological meaning of words: LIWC and computerized text analysis methods. Journal of language and social psychology, 29(1), 2454.

Teo, Thomas. Encyclopedia of critical psychology. Springer Reference, 2014.

Thelwall, M., Buckley, K., Paltoglou, G., Cai, D., \& Kappas, A. (2010). Sentiment strength detection in short informal text. Journal of the American Society for Information Science and Technology, 61(12), 2544-2558.

Tupes, E. C., \& Christal, R. E. (1992). Recurrent personality factors based on trait ratings. Journal of personality, 60(2), 225-251.

Wald, R., Khoshgoftaar, T. M., Napolitano, A., \& Sumner, C. (2012). Using Twitter content to predict psychopathy. In 11th International Conference on Machine Learning and Application (ICMLA) (pp. 394-401). IEEE.

Watt, B. D., \& Brooks, N. S. (2012). Self-report psychopathy in an Australian community sample. Psychiatry, Psychology and Law, 19, 389-401.

Whissell, C. (1989). The dictionary of affect in language. In R. Plutchik \& H. Kellerman (Eds.), Emotion: theory, research, and experience, Vol. 4 (pp. 113-131). New York: Academic Press.

Widiger, T. A., \& Lynam, D. R. (1998). Psychopathy and the five-factor model of personality. Psychopathy: Antisocial, criminal, and violent behavior, 171-187. 
Widom, C. S. (1977). A methodology for studying noninstitutionalized psychopaths. Journal of consulting and clinical psychology, 45(4), 674.

Wiebe, J., Wilson, T., \& Cardie, C. (2005). Annotating expressions of opinions and emotions in language. Language resources and evaluation, 39(2), 165-210.

Williams, K. M., Paulhus, D. L., Hare, R. D. (2007). Capturing the four-factor structure of psychopathy in college students via self-report. Journal of Personality Assessment, 88(2), $205-219$.

Wilson, K., Juodis, M., \& Porter, S. (2011). Fear and loathing in psychopaths: A meta-analytic investigation of the facial affect recognition deficit. Criminal Justice and Behavior, 38(7), 659-668.

Yang, C., Lin, K. H. Y., \& Chen, H. H. (2007, November). Emotion classification using web blog corpora. In Web Intelligence, IEEE/WIC/ACM International Conference on Web Intelligence, 275-278.

Yarkoni, T. (2010). Personality in 100,000 words: A large-scale analysis of personality and word use among bloggers. Journal of research in personality, 44(3), 363-373.

Zhu, J., Wang, H., Tsou, B. K., \& Zhu, M. (2009). Multi-aspect opinion polling from textual reviews. In Proceedings of the 18th ACM conference on Information and knowledge management (pp. 1799-1802). ACM.

Zuckerman, M. (1989). Personality in the third dimension: A psychobiological approach. Personality and individual differences, 10(4), 391-418. 


\section{Appendices}

\section{Appendix A: Interview Questions}

1. How old are you?

2. What is your ethnic background?

3. What year and program are you in?

4. What was high school like for you?

5. How would your teachers describe you in high school?

6. How would other students describe you in high school?

7. How are you finding university so far?

8. If you don't do well on an exam, what is the cause usually and why?

9. What would you like to do after graduating?

10. Are you worried about your future?

11. Have you done any volunteer or paid work?

12. Describe your most recent volunteer or paid job.

13. How have your bosses described your past performances?

14. What were your bosses like?

15. Do you have any close friends?

16. What do you usually do when you hang out with your friends?

17. In your opinion, what's the difference between a friend and an acquaintance?

18. What advice would you give to a friend who was insulted by someone?

19. What advice would you give to a friend who was hit by someone?

20. What is your opinion on the use of alcohol?

21. What is your opinion on the use of marijuana? 
22. What is your opinion on the use of other drugs?

23. What is your opinion on physical fights?

24. Are there any circumstances under which you think it is alright for a person to commit a crime?

25. What personal motives do you think usually causes someone to commit a crime?

26. What or who do you think is largely to blame for someone's criminal activity?

27. Why do you think someone would use verbal threats to control someone?

28. Why do you think someone would use physical threats to control someone?

29. What is your opinion on hurting animals?

30. Do you date?

31. What is attractive to you in a significant other?

32. What is the longest you've gone out with someone?

33. What was that person like?

34. What advice would you give to a friend who's significant other cheated on them.

35. How good are you at getting what you want from friends, family or partners?

36. What do you do when you really want something?

37. How often do you lie to your friends, family or partners?

38. What do you lie about?

39. What do you like to do in your spare time?

40. Are you often bored?

41. What bores you the most?

42. When you have to make a decision, how long do you think about the pros and cons?

43. Has anyone ever said you had a high opinion of yourself? 
44. What's the saddest you've ever felt?

45. What's the happiest you've ever felt?

46. What would you say are your main failures?

47. What are your main accomplishments?

48. What would you say is your biggest problem?

49. What do you consider your worst qualities?

50. What do you consider your best qualities?

51. Describe one of your worst memories.

52. Describe one of your best memories.

53. Tell me about your family.

54. What was life like growing up?

55. How would you describe your parents?

56. Do your parents get along?

57. Do you have any siblings?

58. How is your relationship with your siblings?

59. How strict were things in your home while growing up?

60. Is there anything else you would like to add? 


\section{Appendix B: SRP-III-SF Questionnaire}

Please rate the degree to which you agree with the following statements. You can be honest because your name will be detached from the answers as soon as they are submitted.

\begin{tabular}{|c|c|c|c|c|}
\hline 1 & 2 & 3 & 4 & 5 \\
\hline Disagree Strongly & Disagree & Neutral & Agree & Agree \\
& & & & Strongly \\
\hline
\end{tabular}

1. I'm a rebellious person

2. I have never been involved in delinquent gang activity.

3. Most people are wimps.

4. I've often done something dangerous just for the thrill of it.

5. I have tricked someone into giving me money.

6. I have assaulted a law enforcement official or social worker.

7. I have pretended to be someone else in order to get something.

8. I like to see fist-fights.

9. I would get a kick out of 'scamming' someone.

10. It's fun to see how far you can push people before they get upset.

11. I enjoy doing wild things.

12. I have broken into a building or vehicle in order to steal something or vandalize

13. I don't bother to keep in touch with my family any more.

14. I rarely follow the rules.

15. You should take advantage of other people before they do it to you.

16. People sometimes say that I'm cold-hearted....

17. I like to have sex with people I barely know.

18. I love violent sports and movies.

19. Sometimes you have to pretend you like people to get something out of them

20. I was convicted of a serious crime.

21. I keep getting in trouble for the same things over and over. 
22. Every now and then I carry a weapon (knife or gun) for protection.

23. You can get what you want by telling people what they want to hear.

24. I never feel guilty over hurting others.

25. I have threatened people into giving me money, clothes, or makeup.

26. A lot of people are "suckers" and can easily be fooled.

27. I admit that I often "mouth off" without thinking.

28. I sometimes dump friends that I don't need any more.

29. I purposely tried to hit someone with the vehicle I was driving. 